\title{
Proteostasis Regulation in the Endoplasmic Reticulum: An Emerging Theme in the Molecular Pathology and Therapeutic Management of Familial Hypercholesterolemia
}

\author{
Deepu Oommen ${ }^{1 \dagger}$, Praseetha Kizhakkedath ${ }^{1 \dagger}$, Aseel A. Jawabri ${ }^{1}$, Divya Saro Varghese ${ }^{1}$ \\ and Bassam R. Ali, ${ }^{1,2,3 *}$
}

${ }^{1}$ Department of Genetics and Genomics, College of Medicine and Health Sciences, United Arab Emirates University, Al-Ain, United Arab Emirates, ${ }^{2}$ Department of Pathology, College of Medicine and Health Sciences, United Arab Emirates University, Al-Ain, United Arab Emirates, ${ }^{3}$ Zayed Center for Health Sciences, College of Medicine and Health Sciences, United Arab

OPEN ACCESS

Edited by:

Uma Ramaswami,

Royal Free London NHS Foundation

Trust, United Kingdom

Reviewed by:

Gerarda Cappuccio,

University of Naples Federico II, Italy Nicole Ziliotto,

University of Milano Bicocca, Italy

*Correspondence:

Bassam R. All

bassam.ali@uaeu.ac.ae

${ }^{\dagger}$ These authors have contributed equally to this work

Specialty section:

This article was submitted to Genetics of Common and Rare

Diseases,

a section of the journal

Frontiers in Genetics

Received: 07 June 2020

Accepted: 31 August 2020

Published: 23 September 2020

Citation:

Oommen D, Kizhakkedath $P$, Jawabri $A A$, Varghese $D S$ and Ali $B R$

(2020) Proteostasis Regulation in the Endoplasmic Reticulum: An Emerging Theme in the Molecular

Pathology and Therapeutic Management of Familial

Hypercholesterolemia.

Front. Genet. 11:570355.

doi: 10.3389/fgene.2020.570355
Familial hypercholesterolemia $(\mathrm{FH})$ is an autosomal genetic disease characterized by high serum low-density lipoprotein (LDL) content leading to premature coronary artery disease. The main genetic and molecular causes of $\mathrm{FH}$ are mutations in low-density lipoprotein receptor gene $(L D L R)$ resulting in the non-clearance of $L D L$ from the blood by hepatocytes and consequently the formation of plaques. LDLR is synthesized and glycosylated in the endoplasmic reticulum (ER) and then transported to the plasma membrane via Golgi. It is estimated that more than $50 \%$ of reported $\mathrm{FH}$-causing mutations in LDLR result in misfolded proteins that are transport-defective and hence retained in ER. ER accumulation of misfolded proteins causes ER-stress and activates unfolded protein response (UPR). UPR aids protein folding, blocks further protein synthesis, and eliminates misfolded proteins via ER-associated degradation (ERAD) to alleviate ER stress. Various studies demonstrated that ER-retained LDLR mutants are subjected to ERAD. Interestingly, chemical chaperones and genetic or pharmacological inhibition of ERAD have been reported to rescue the transport defective mutant LDLR alleles from ERAD and restore their ER-Golgi transport resulting in the expression of functional plasma membrane LDLR. This suggests the possibility of pharmacological modulation of proteostasis in the ER as a therapeutic strategy for $\mathrm{FH}$. In this review, we picture a detailed analysis of UPR and the ERAD processes activated by ER-retained LDLR mutants associated with $\mathrm{FH}$. In addition, we discuss and critically evaluate the potential role of chemical chaperones and ERAD modulators in the therapeutic management of $\mathrm{FH}$.

Keywords: ERAD pathway, cholesterol, familial hypercholesterolemia, Class II mutations, lipid metabolism, LDLR, ER stress, unfolded protein response

\footnotetext{
Abbreviations: CAD, coronary artery disease; CMV, cytomegalovirus; ERAD, endoplasmic reticulum associated degradation; ER, endoplasmic reticulum; FH, familial hypercholesterolemia; LDLR, low-density lipoprotein receptor; UPR, unfolded protein response.
} 


\section{INTRODUCTION}

\section{Familial Hypercholesterolemia}

Familial hypercholesterolemia (FH) is a genetic disorder that results in altered lipid metabolism and consequently leading to elevated levels of plasma low-density lipoprotein cholesterol (LDL-C) (Soutar and Naoumova, 2007). Clinically, FH is characterized by increased levels of LDL-C, tendon xanthomas, corneal arcus, and premature coronary artery diseases (CAD) such as atherosclerosis (Müller, 1938; Kawaguchi et al., 1999; Kitahara et al., 2019). Mutations in the low-density lipoprotein receptor gene $(L D L R)$ account for more than $80 \%$ of monogenic FH (Brown et al., 1986) (FHCL1, OMIM\#143890). Monogenic $\mathrm{FH}$ can also be caused by mutations in other genes including APOB (FHCL2, OMIM\#144010) (Innerarity et al., 1990), PCSK9 (FHCL3, OMIM\#603776) (Abifadel et al., 2003), and LDLRAP1 (FHCL4, OMIM\# 603813) (Garcia et al., 2001). FH can exist in both heterozygous and homozygous forms with homozygous $\mathrm{FH}(\mathrm{HoFH})$ patients at far greater risk of developing CAD in their first decade of life (Alonso et al., 2014). The clinical manifestations of a homozygous patient suffering from FH begin in the first decade of their life including abnormal cholesterol storage which results in the appearance of cutaneous xanthomas and the appearance of tendon xanthomas particularly in the joints and fingers. Another late symptom is the manifestation of xanthelasmata as well as corneal arcus. Also, coronary manifestations in HoFH appear in their second and third decades (Klose et al., 2014) though fatal myocardial infarctions (MIs) are possible even in early childhood (Wiegman et al., 2015). On the other hand, the clinical manifestations in heterozygous $\mathrm{FH}$ patients are possible from early adulthood onward and premature $\mathrm{CAD}$ in the second or third decade of life. Sometimes symptoms may remain clinically hidden (Klose et al., 2014). If left untreated, approximately $50 \%$ heterozygous males and $15 \%$ females have a fatal MI by the age of 60 (Henderson et al., 2016). In recent studies, it has been shown that the prevalence of heterozygous FH has increased and affects between 1:200 or 1:300 in most populations (Nordestgaard et al., 2013).

Cholesterol is an essential component of membranes and serves as a precursor for steroid molecules such as hormones, bile acids and vitamin $\mathrm{D}$. Cellular cholesterol requirement is met either by de novo intracellular synthesis or by uptake of dietary cholesterol (Goldstein and Brown, 1990). Receptor-mediated endocytosis of cholesterol mediated by LDLR, unraveled by the seminal work of Brown and Goldstein, is the main pathway for cellular uptake of exogenous cholesterol (Brown et al., 1986). On the cell membrane, the LDLR receptors are localized to clathrin-coated pits and when the LDL-bound cholesterol attaches to the receptor, the complex is internalized and fuse with early sorting endosomes. There the receptor dissociates from the lipid and recycles back to the cell-surface repeating this cycle every $10 \mathrm{~min}$ (Brown et al., 1986). The LDL particles are eventually delivered via endosomal trafficking to the lysosomes for degradation and the cholesterol is released within the cell. Excess cellular cholesterol is esterified and stored in lipid droplets in the endoplasmic reticulum (ER) (Ikonen, 2008). Cellular cholesterol homeostasis is a tightly regulated process and the ER plays a crucial role in cholesterol sensing, regulation, and synthesis (Röhrl and Stangl, 2018). The ER is also the site of synthesis of many membrane proteins including that of LDLR which is in turn subject to feedback regulation by intracellular cholesterol levels. The review aims to present how LDLR mutants implicated in FH deregulates ER homeostasis and also explores the possibilities of targeting ER-proteostasis machinery for therapeutic management of $\mathrm{FH}$.

\section{Low-Density Lipoprotein Receptor (LDLR): Gene, Protein Structure, and Function}

The low-density lipoprotein receptor (LDLR) is the prototype receptor of a group of structurally and functionally similar cell surface receptors. LDLR is encoded by the LDLR gene located on chromosome 19p13.1-13.3. It spans $\sim 45 \mathrm{~kb}$ and comprises 18 exons that are translated into 860 amino acids including a signal sequence of 21 amino acids which is cleaved during translocation into the ER (Francke et al., 1984) (Figure 1A). Each exon or group of exons constitutes a particular domain in the LDLR (Figure 1A) (Gent and Braakman, 2004). There are five LDLR domains and each domain mediates a specific function (Klee and Zimmermann, 2019) which are: a ligand-binding domain (LBD), an epidermal growth factor (EGF) homology domain, an $O$-linked sugar region, a membrane-spanning domain and a $C$-terminal cytoplasmic tail domain (Gent and Braakman, 2004) (Figure 1B).

The LBD is made up of 292 amino acids and consists of seven cysteine-rich ligand-binding repeats (LRs) each composed of 40 amino acid residues (Yamamoto et al., 1984; Südhof et al., 1985; Fass et al., 1997). Six cysteine residues along with a group of negatively charged amino acids in the LR sequence interact with positively charged residues on the APOB and APOE molecules to mediate the recognition and binding of LDL-C to LDLR (Bradley and Gianturco, 1986). The second domain is the EGF precursor domain which is composed of 400 amino acids and contributes to the dissociation of the LDL particles from the LDLR-LDL complex in the endosome at a low pH (Davis et al., 1987; Rudenko et al., 2002). The EGF precursor domain is composed of three EGF-like repeats, EGF-like 1, 2, and 3 each consisting of $\sim 40$ amino acids. A domain of six YWTD motifs known as $\beta$-propeller domain occurs between repeats 2 and 3 (Springer, 1998; Jeon et al., 2001) (Figure 1B).

The $O$-linked sugars domain plays a major role in the posttranslational modification of LDLR. This domain is encoded by exon 15 and is composed of 48 amino acids consisting of 18 threonine and cysteine residues that act as attachment sites for $O$-linked sugar chains. The membrane-spanning domain is responsible for LDLR integration and attachment to the cell membrane (Russell et al., 1984; Yamamoto et al., 1984; Südhof et al., 1985). Endocytosis of LDLR-LDL complex is mediated by the 5th domain in the LDLR which is the cytoplasmic tail encoded by exon 17 and exon 18 (Goldstein and Brown, 2009). 
A

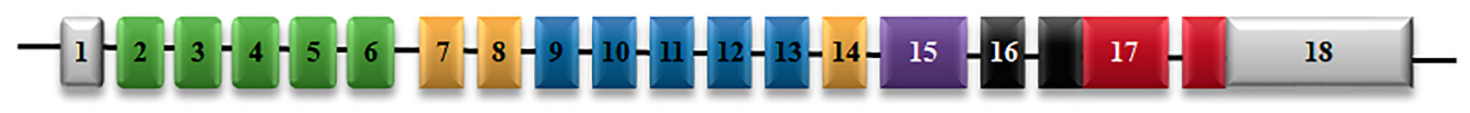

B

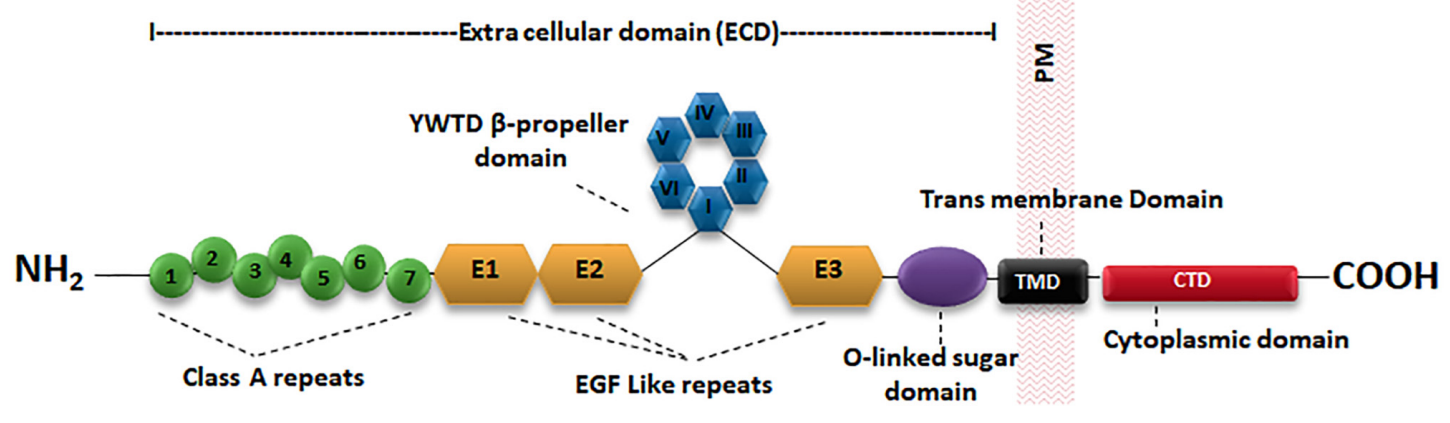

C

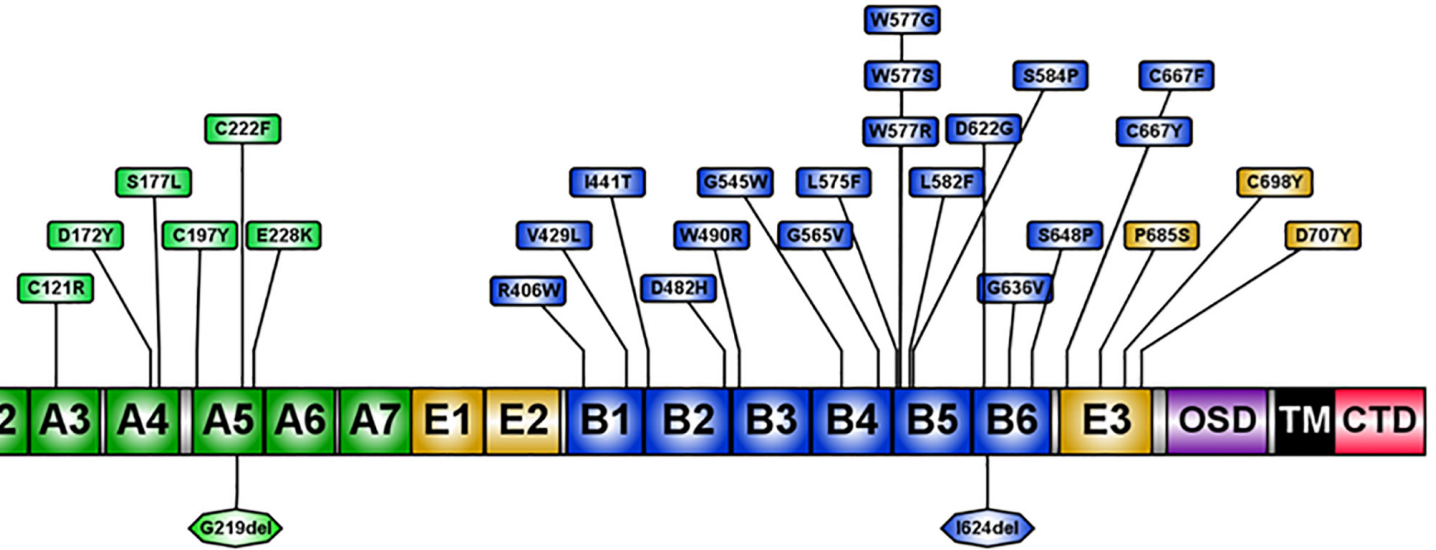

FIGURE 1 | Schematic representation of the LDLR gene and protein structure. (A) The 18 exons of $L D L R$ are numbered and exons coding for different domains of the LDLR protein are represented by different colors. (B) The LDLR protein has an extracellular domain (ECD), a membrane-spanning domain (TMD) and a cytoplasmic C-terminal domain. The ECD consists of a ligand-binding domain (LBD), an epidermal growth factor (EGF) homology domain and an O-linked sugar region. The EGF homology domain is composed of three EGF-like repeats 1-3 and a $\beta$-propeller domain of six YWTD motifs occurs between repeats 2 and 3 . (C) Schematic diagram showing the amino acid positions of well known class II mutations (Table 2) and the substitutions are marked in boxes. OSD, O-linked sugar domain.

\section{LDLR Mutations-Types and Classifications}

A total of 2,299 variants have been reported in association with $\mathrm{FH}$ in the Human Gene Mutation Database (HGMD) (Stenson et al., 2017), ranging between missense mutations, nonsense mutations, deletions, insertions or duplications. Based on functional consequences, LDLR mutations have been classified into five major classes (Hobbs et al., 1992), as described in detail in Table 1. Briefly, the functional impact of each classes are, Class I: Defects in synthesis of LDLR mainly due to null alleles; Class II: Impaired trafficking of the LDLR to Golgi compartments and cell surface, due to improper folding and complete or partial retention in the ER (2A and $2 \mathrm{~B}$, respectively); Class III: Deficient in binding to ligands; Class IV: Impaired clustering and endocytosis of ligand-bound receptors; Class V: Interferes with the cell-surface recycling of internalized LDLR due to defects in dissociation of ligand from the receptor, subsequently leading to the degradation of the receptor in the lysosome (Beglova et al., 2004; Van Hoof et al., 2005). Class VI is a new functional class of LDLR variants where the LDLR is properly synthesized by the ER and Golgi apparatus but fail to undergo basolateral sorting in polarized cells (Koivisto et al., 2001). Additional novel functional classes are emerging with increasing functional data (Susan-Resiga et al., 2017) and most recently a novel class of LDLR variants inducing ectodomain cleavage of the LDL receptor in the ER has been suggested (Strøm et al., 2014, 2017).

Around $50 \%$ of reported LDLR mutations are Class II mutants which are implicated to be transport-defective (Varret and Rabès, 2012). At present, there are 895 missense mutations reported in the HGMD (Stenson et al., 2017), occurring at 451 amino acid codons distributed across the whole length of the protein (Supplementary Figure S1). However, only limited information is available on the functional classes of these variants (BenitoVicente et al., 2018). A compilation of functionally validated Class II mutations from the published literature is presented in Table 2. 
TABLE 1 | Classes of LDLR variants.

\begin{tabular}{|c|c|c|}
\hline $\begin{array}{l}\text { LDLR variant } \\
\text { classes }\end{array}$ & Type of variants & Protein/functional impact \\
\hline Class 1 & $\begin{array}{l}\text { - Early stop codons } \\
\text { - Mutations in the promoter regions } \\
\text { - Splicing aberrations } \\
\text { - Large exonic deletions }\end{array}$ & Synthesis defective: Defects in LDLR protein synthesis \\
\hline $\begin{array}{l}\text { Class II: } \\
\text { - Class II A } \\
\text { - Class II B }\end{array}$ & $\begin{array}{l}\text { - Missense mutations in the cysteine-rich domains } \\
\text { - In-frame deletions/duplications } \\
\text { - Protein truncating mutations }\end{array}$ & $\begin{array}{l}\text { Transport defective: Defects in LDLR folding, maturation and transport } \\
\text { in the secretory pathway } \\
\text { Class IIA: Completely retained in the ER due to folding defects } \\
\text { Class II B: Transport-competent but ER-retained due to slower } \\
\text { processing }\end{array}$ \\
\hline Class III & - Point mutations clustering in the ligand binding domain & Binding defective: Transport-competent but defective in binding to LDL \\
\hline Class IV & $\begin{array}{l}\text { - Mutations in the 4th and 5th domains } \\
\text { - Complete deletion of those LDLR domains }\end{array}$ & $\begin{array}{l}\text { Clustering and endocytosis defective: Impair with the clustering of } \\
\text { ligand-bound LDLR in clathrin coated pits and endocytosis of } \\
\text { LDLR-LDL complex }\end{array}$ \\
\hline Class V & - Deletions in the EGF precursor domain & $\begin{array}{l}\text { Dissociation and recycling defective: The LDLR-LDL complex is } \\
\text { successfully internalized in the cell, but dissociation of the LDLR from } \\
\text { the LDL does not happen leading to the degradation of LDLR along } \\
\text { with LDL in the lysosomes (Beglova et al., 2004; Van Hoof et al., 2005) }\end{array}$ \\
\hline
\end{tabular}

A schematic representation of the position of occurrence of the reported variants is shown in Figure 1C. Unlike the other classes of mutants that interfere with a specific function of the receptor, class II mutations cause global conformation defects leading to their retention in the ER, potentially overwhelming the cellular proteostasis machinery in addition to impaired cholesterol homeostasis (Gent and Braakman, 2004).

\section{MECHANISMS OF PROTEIN QUALITY CONTROL AND PROTEOSTASIS REGULATION IN THE ER}

In eukaryotes, an estimated one-third of all newly synthesized proteins enter the ER to undergo post-translational modifications and achieve their three-dimensional native conformation, before reaching their proper cellular destination (Brodsky and Skach, 2011). However, protein folding is an inherently error-prone process and only a fraction of all produced proteins reaches a native conformation. Multiple stringent quality control mechanisms operates in the ER to ensure that only properly folded proteins are transported out of the ER and protein homeostasis or "proteostasis" is maintained (Sun and Brodsky, 2019). Many membrane and secretory proteins that fail to conform to the ER quality control (ERQC) are dislocated into the cytosol and degraded by the proteasome by a process termed as ER-associated degradation (ERAD) (Vembar and Brodsky, 2008; Ruggiano et al., 2014; Sun and Brodsky, 2019). Misfolded proteins can still retain their function and premature ERAD of mutant misfolded proteins is accounted for the cellular pathogenesis of several congenital disorders (Ward et al., 1995; Hume et al., 2009; Ali et al., 2011; Al-Kindi et al., 2014; Kizhakkedath et al., 2014, 2019; John et al., 2015). Sometimes the quality control mechanisms fail to recognize folding-incompetent forms which leads to the accumulation of folding-intermediates in the ER, causing ER stress. The cells respond to ER stress by initiating the unfolded protein response (UPR), an integrated stress response program, that aims to increase cell's folding capacity, accelerate clearance of unfolded proteins by ERAD, and restore protein homeostasis in the cell (Karagöz et al., 2019). Unresolved ER stress may lead to cell death (Karagöz et al., 2019). The ERretained LDLR class II mutants have been reported to be degraded through a proteasome-mediated pathway (Li et al., 2004) and have been shown to activate ER-stress pathways (Sørensen et al., 2006).

\section{Major Components of ERAD}

ER-associated degradation is a collective term for a succession of events that starts with substrate recognition, followed by chaperone-assisted translocation to the cytosol and culminates in degradation by the ubiquitin-proteasome system (UPS) (Brodsky and Skach, 2011; Sun and Brodsky, 2019). Though complex, the fundamental ERAD machinery is conserved in eukaryotes from yeast to mammals (Brodsky and Skach, 2011; Sun and Brodsky, 2019). The folding of nascent polypeptides entering the ER is assisted by a chaperone system comprising of classical ER chaperones, lectin chaperones and protein disulfide isomerases (PDIs) (Braakman and Hebert, 2013). Classical chaperones belonging to the heat shock proteins (HSPs) family are GRP78/BiP (Hsp70), GRP94 (Hsp90), and J-proteins (Hsp40) (Braakman and Hebert, 2013). GRP78 recognizes and binds to misfolded proteins with exposed hydrophobic residues and helps in interaction other HSP chaperones and PDIs (Ni and Lee, 2007). N-linked glycosylation of Asn-X-Ser/Thr motif is an important post-translational modification that help nascent proteins to remain soluble and prevent aggregation by masking the hydrophobic stretches in the protein (Aebi et al., 2010). $N$-glycosylation involves the attachment of a preassembled 
TABLE 2 | List of all functionally characterized Class || LDLR variants.

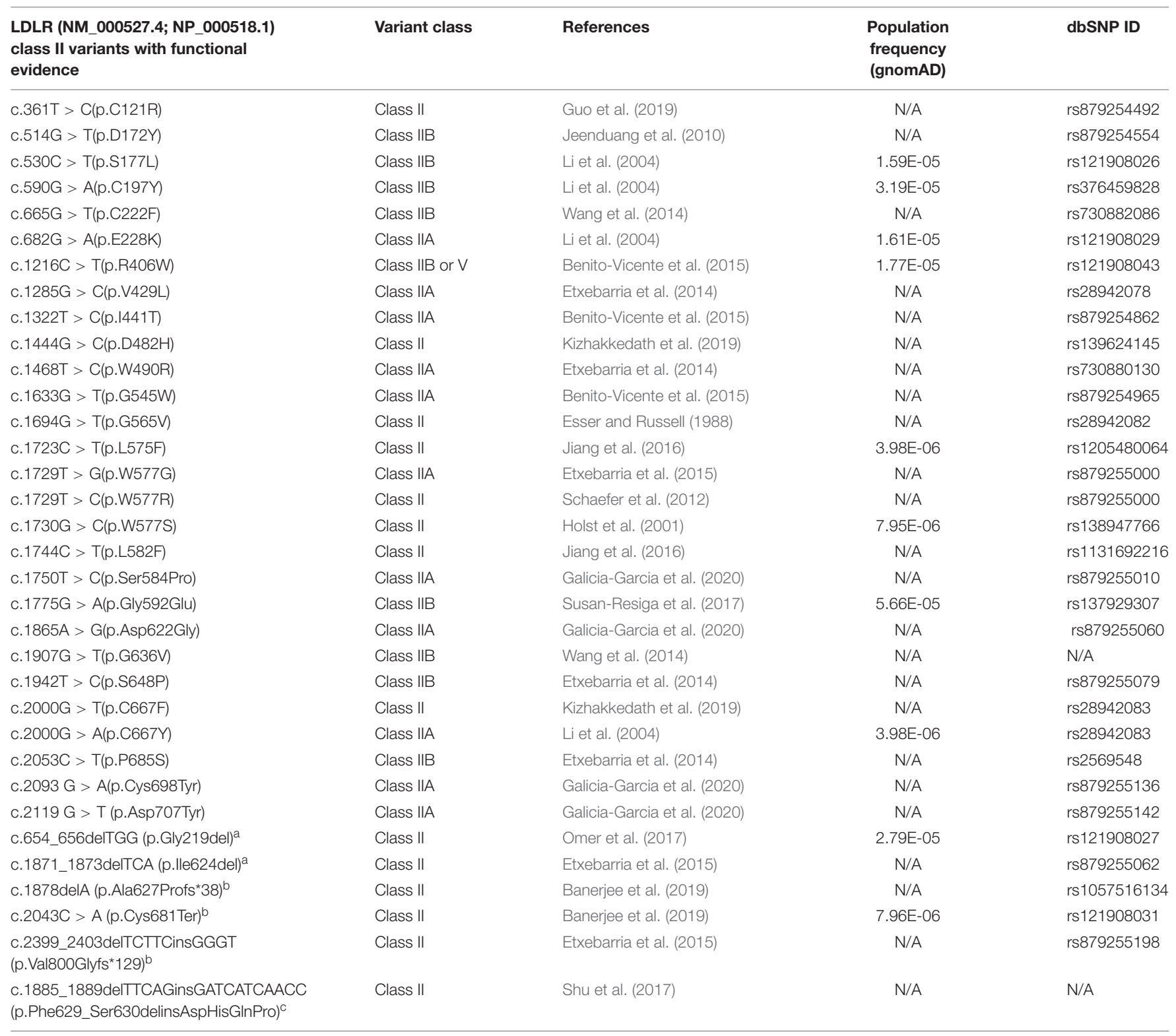

${ }^{a}$ In-frame amino acid deletions. ${ }^{b}$ Protein truncating variant. ${ }^{c}$ Complex deletion-insertion.

carbohydrate, comprised of three glucoses, nine mannoses, and two $\mathrm{N}$-acetyl glucosamines ( $\left.\mathrm{Glc}_{3} \mathrm{Man}_{9} \mathrm{GlcNAc}_{2}\right)$, to the Asn residue (Aebi et al., 2010).

Enzymatic deglucosylation of the N-glycan to Glc $_{1}$ Man $_{9}$ GlcNAc $_{2}$ by glucosidases I (GI) and 2 (GII), makes it a high-affinity ligand for lectin chaperones such as calnexin (CNX) and calreticulin (CRT). Binding of CNX/CRT to glycoproteins facilitates their retention in ER, prevention of aggregation and recruitment of PDIs such as ERp57 (PDIA3) (Zapun et al., 1998; Lamriben et al., 2016). Removal of the final glucose by GII prevents further binding CNX/CRT and if folded, the substrates progress toward ER exit sites. Unfolded proteins undergo further rounds of reglucosylation by UDP-glucose/glycoprotein glucosyltransferase (UGGT) and are reverted to CNX/CRT for folding. If folded the glycoproteins eventually exit the cycle (Lamriben et al., 2016) and terminally misfolded proteins are released from this cycle and diverted to ERAD for disposal. An example of glycoprotein folding is that of LDLR which is represented in Figure 2.

Demannosylation by the ER-resident mannosidases such as ER mannosidase 1 (ERMan1) and ER-degradation enhancing mannosidase-like proteins (EDEM1, 2, and 3) results in $N$-glycans with deglucosylated, demannosylated forms (Man5-Man7) that are incompatible with UGGTmediated reglucosylation (Shenkman and Lederkremer, 2019). The deglycosylated and demannosylated misfolded proteins are selectively captured by the mannose-specific lectins (OS9 and XTP-3B) for their further delivery to the 


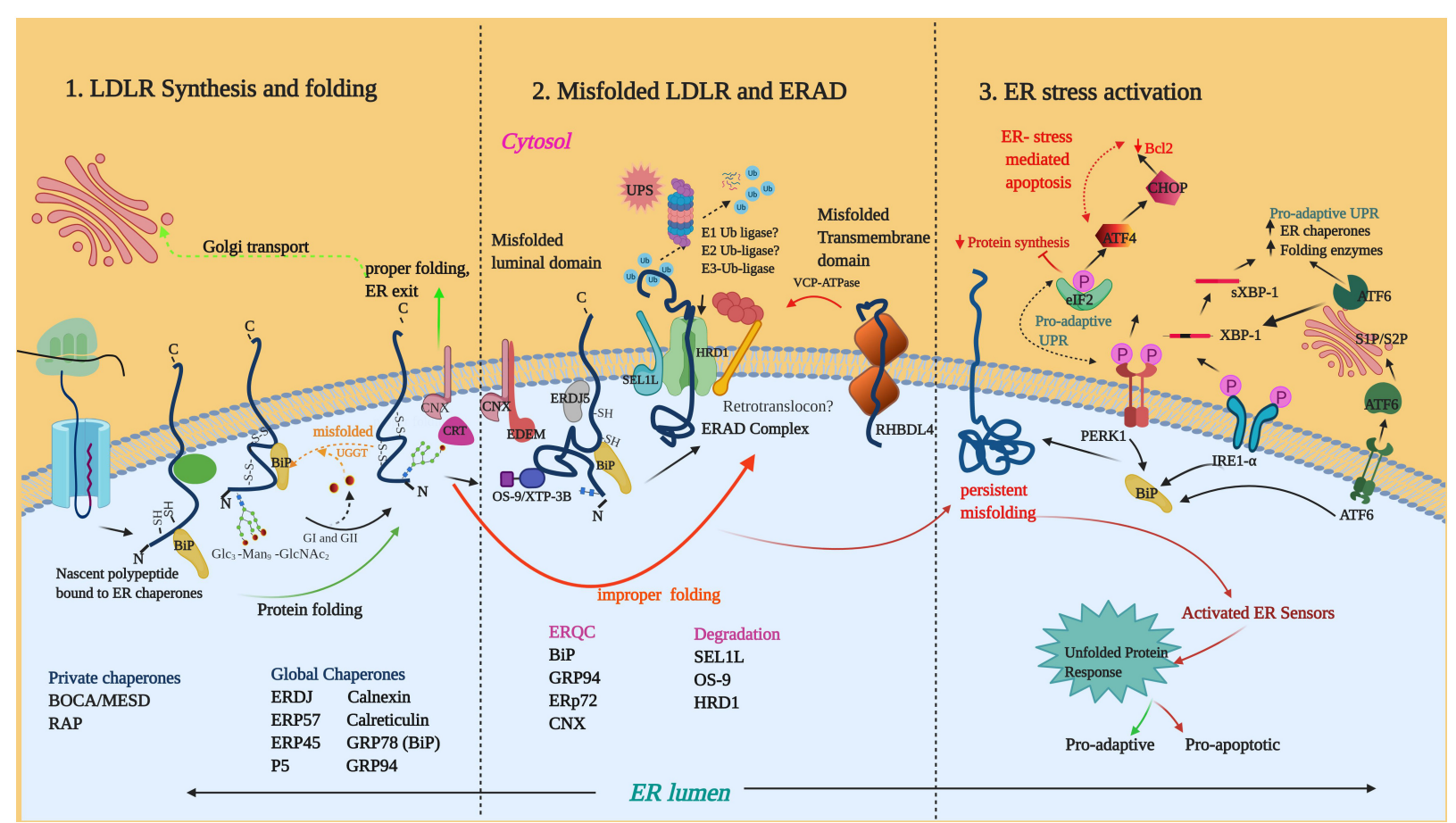

FIGURE 2 | LDLR folding, misfolding and activation of UPR: (1) The nascent LDLR is co-translationally inserted into the ER membrane and the LDLR ectodomain undergoes folding in the ER lumen with the assistance of several global and private chaperones as listed in the figure. (2) Misfolded proteins such as Class II mutants engage in prolonged interaction with the chaperone system. BiP, GRP94, ERP72 are ERQC factors implicated in LDLR retention. Terminally misfolded proteins are extracted from the chaperone system and delivered to membrane-embedded ERAD complex for degradation by the ubiquitin-proteasome system. So far, the components known to be involved in LDLR-ERAD are OS9, SEL1L and HRD1. RHBDL4 is a metalloprotease involved in the ERQC of ERAD-M candidates of LDLR. (3) Accumulation of misfolded LDLR induces ER stress and activates the UPR proteins IRE1, PERK, and ATF6. Phosphorylation of elF2 $\alpha$ by PERK leads to the attenuation of protein translation. Activated IRE1 $\alpha$ induces splicing of the long XBP1 mRNA to form XBP1s mRNA which encodes XBP1s protein. Activated ATF6 is cleaved in the Golgi to form the active ATF6 N-terminal fragment. XBP1s and ATF6 are transcription factors that target the transcriptional induction of UPR target genes. Unresolved ER-stress turn-on proapoptotic pathways through the PERK-arm of the UPR. Illustration created with Biorender.com.

ER degradation machinery (Olzmann et al., 2013) (Figure 2). Non-glycosylated misfolded proteins are also targeted for ERAD and it is believed that features other than glycan trimming may contribute to their recognition (OkudaShimizu and Hendershot, 2007). Non-glycosylated proteins can be directly recruited by BiP (GRP78) and J-domain proteins to ERAD complex, bypassing the CNX pathway (Ushioda et al., 2013).

Once selected, the ERAD substrates are delivered to the cytosol for ubiquitination by E3-ubiquitin ligases and proteolytic degradation by the UPS. In yeast, specialized ERAD pathways exist to degrade misfolded proteins with defects exposed in the ER luminal (ERAD-L), transmembrane (ERAD-M), and cytosolic domains (ERAD-C) (Carvalho et al., 2006). An emerging body of evidence suggests that mammalian ERAD does not follow rigid rules for substrate engagement and an array of E3 ligases cooperates to complete the ERAD processing of substrates with diverse topologies (Christianson et al., 2011; Olzmann et al., 2013). Two well-known mammalian E3 ubiquitin ligases are polytopic RING domain ubiquitin ligases, HMG-CoA reductase degradation protein 1 (HRD1/SYVN1) and gp78/autocrine motility factor receptor (AMFR) (Fang et al., 2001; Nadav et al., 2003). E3 ligases such as RMA1 (RNF5),
TRC8, TEB4 (MARCH IV) have been reported to be involved in the degradation of a limited number of ERAD clients (Olzmann et al., 2013).

Since the proteins targeted for degradation have diverse structures and topologies, distinct combinations of adaptors that recognize these features are recruited by the E3-ubiquitin ligases. In mammals, the transmembrane (TM) protein SEL1L works in conjunction with the HRD1 E3 ligase and is necessary to deliver the ERAD substrates from ER lectins (OS9, XTP) to HRD1 (Christianson et al., 2008; Hosokawa et al., 2008). Depletion of SEL1L has been reported to destabilize HRD1 and prevent the degradation of misfolded lumenal/TM proteins (Christianson et al., 2008; Horimoto et al., 2013; Bianchini et al., 2014; Kizhakkedath et al., 2018). Other mammalian ER adapters are ERLINSs, INSIGs and F-box proteins (Olzmann et al., 2013).

Derlin family of proteins DER1, 2, and 3 have been proposed to play a role in substrate dislocation through association with HRD1/SEL1L (Lilley and Ploegh, 2005). During dislocation, the disulfide bonds are reduced by oxidoreductase enzymes like ERFAD and ERDJ5 (Smith et al., 2011) and partially unfolded by rhomboid pseudoproteases such as Derlins, UBCA2 and UBXD8 (Olzmann et al., 2013). The 
dislocation process is powered by the cytosolic valosin containing protein (VCP)/p97 ATPase (Guerriero and Brodsky, 2012). During dislocation, an E1 ubiquitin ligase enzyme activates ubiquitin and an ubiquitin-conjugating enzyme (E2 ligase) in conjunction with a ubiquitin ligase (E3) then transfer ubiquitin to the substrate (Christianson and Ye, 2014). UBA1 is a well characterized E1 ligase enzyme in humans and initially believed to be the only subtype of E1 ligases. Examples of mammalian E2 ligases are UBE2J1, UBE2J2 and UBE2G2. The ubiquitin-tagged substrates are then delivered to degradation by the $26 \mathrm{~S}$ proteasome in the cytosol with or without the help of small cytosolic heat-shock chaperones (Christianson and Ye, 2014) (Figure 2).

\section{Quality Control of Membrane Proteins With Defective Transmembrane Domains}

The quality control of TM proteins containing defects in their membrane-spanning domain appear to be distinct from that of ERAD-L and ERAD-C substrates, since luminal or cytosolic factors do not have access to the domain location. An intramembrane rhomboid protease, RHBDL4 has been shown to cleave TM-anchors of unstable single-membrane spanning or polytopic membrane proteins in an ubiquitin-dependent manner and divert them to the UPS coupled to VCP/p97 ATPase (Figure 2) (Fleig et al., 2012). Recent studies have shown that the ERAD-M substrates containing less hydrophobic TM-domains get translocated entirely to the ER lumen leading to recognition by $\mathrm{BiP}$ and degradation through the canonical ERAD-L pathway (Feige and Hendershot, 2013). Another study proposed that degradation of an ERAD-M substrate containing an unassembled TMD was dependent upon ubiquitination on cytoplasmic lysine residues and occurs through a specific ERAD pathway that is mechanistically distinct from that which mediates degradation of membrane proteins with luminal folding defects (Briant et al., 2015).

\section{Non-canonical ERAD Pathways}

While ERAD is highly efficient in handling a variety of misfolded proteins, some membrane and soluble proteins form aggregates and place constraints on ER retrotranslocation machinery. These aggregates are diverted to the lysosome for degradation via alternative pathways collectively termed as ER-to-lysosomeassociated degradation (ERLAD) (Houck et al., 2014; Fregno and Molinari, 2019; De Leonibus et al., 2019). ERLAD include (i) ER-phagy, (ii) microautophagy, and (iii) vesicular transport (De Leonibus et al., 2019; Fregno and Molinari, 2019). In ER-phagy, ER fragments are engulfed by a double membrane LC3-decorated autophagosome that fuses with the lysosome. Microautophagy is an ER autophagy where misfolded proteins segregated on ER exit sites (ERES) coated with LC3 and COPII, are directly engulfed by lysosomal invagination or protrusion. Vesicular transport is mediated by single membrane ER-derived vesicles that bud from the ER and fuse with endolysosomes decorated with LC3 (De Leonibus et al., 2019). In ER-phagy, membrane-embedded LC3-binding receptors regulate the delivery of ER-subdomains to lysosome. In mammals the
ER-phagy receptors include FAM134B, RTN3, SEC62, CCPG1, ATL3, and TEX264 (Khaminets et al., 2015).

\section{ERAD PROCESSING OF LDLR MUTANTS}

\section{Canonical Role of ERAD in the Regulation of Sterol Synthesis}

Other than performing as a quality control checkpoint, ERAD plays a quintessential role in providing protein quantity control as well in response to environmental demands (Hegde and Ploegh, 2010; Printsev et al., 2017). Cholesterol metabolism and homeostasis are tightly regulated processes and ubiquitin-dependent protein degradation is involved in transcriptional regulation, the synthesis, efflux and uptake of cholesterol (Sharpe et al., 2014). At the transcriptional level, cholesterol metabolism is regulated by the opposing actions of two transcription factors, namely sterol regulatory element-binding proteins (SREBPs) and the liver $\mathrm{X}$ receptors (LXRs) (Sharpe et al., 2014). Under low cellular cholesterol levels, SREBPs are involved in the transcriptional induction of genes required for de novo biosynthesis of cholesterol and LDLR for the uptake of cholesterol (Innerarity et al., 1990). Under elevated cellular cholesterol levels, LXRs induce genes involved in cholesterol efflux pathways and degradation of LDLR (Nadav et al., 2003).

The most widely known example of quantity control by ERAD is the post-translational feedback-regulation of 3-hydroxy3-methyl-glutaryl-CoA reductase (HMGCR), a rate-limiting enzyme in the mevalonate pathway which produces cholesterol and other isoprenoids (DeBose-Boyd, 2008). The accumulation of sterols in ER membranes triggers the binding of HMGCR to ER-membrane proteins INSIG1 and INSIG2 which in turn recruit ubiquitin ligases GP78, TRC8, and RNF145 (Jo et al., 2011; Menzies et al., 2018). Ubiquitinated reductase is then extracted by VCP ATPase and delivered to the proteasome (Jo et al., 2011). Squalene monooxygenase/Epoxidase (SQLE) is another rate-limiting enzyme in the mevalonate pathway downstream of HMGCR and recent studies have shown that another ER-resident E3 ligase, MARCH6 is involved in the ubiquitin-proteasome degradation of SQLE (Loregger et al., 2015). MARCH6 is postulated to play a multifaceted role in cholesterol homeostasis as an endogenous negative modulator of SREBP and HMGCR (Loregger et al., 2015). The E3 ligases FBW7 and RNF20 are involved in the ubiquitin-dependent regulation of SREBPs (Sundqvist et al., 2005; Lee et al., 2014; Sharpe et al., 2014). Inhibition of cholesterol synthesis activates SREBP and transcriptional upregulation of LDLR.

Other than transcriptional regulation by SREBP, LDLR is post-translationally regulated by ubiquitin-dependent degradation mediated by the E3 ligase- inducible degrader of the LDLR (IDOL) (Zelcer et al., 2009). IDOL is transcriptionally controlled by LXRs and appear to preferentially ubiquitinate the cytoplasmic tails of plasma-membrane localized LDLR and mediate lysosomal rather than proteasomal degradation of the receptor (Zelcer et al., 2009). Interestingly, IDOL was found to be capable of regulating the ER located LDLR forms also, since a 
Class II LDLR mutant G546D was demonstrated to be degraded by IDOL by a lysosomal pathway (Zelcer et al., 2009).

\section{Molecular Players in the LDLR Folding Pathway}

The LDLR receptor family has a modular organization consisting of LDL-repeats, EGF-like repeats with $\beta$-propeller, a single TM domain, and a small cytosolic tail (Figure 1B) (Gent and Braakman, 2004). Even though the different domains are organized from $\mathrm{NH}_{2}$-to- $\mathrm{COOH}$ terminus and the folding is co-translational, the nascent LDLR polypeptide is demonstrated to fold rapidly into compact structures by forming non-native disulfide bonds linking distant domains of the receptor (Jansens et al., 2002; Gent and Braakman, 2004). The non-native disulfides are later isomerized and native short-range disulfide bridges are formed with high efficiency and rarely lead to aggregate formation. The high-efficiency folding of LDLR requires the assistance of several general and private chaperones (Garcia et al., 2001). The HSP chaperone GRP78 and PDI family member ERDJ5 have been reported to be involved in the folding of LDLR (Gent and Braakman, 2004; Oka et al., 2013).

ERDJ5 (DNAJC10) is an ER-localized oxidoreductase containing J domain and thioredoxin domains important for its disulfide exchange activity (Oka et al., 2013). ERDJ5 is known to participate in the degradation pathway of misfolded proteins by reducing the disulfide bonds prior to retrotranslocation (Ushioda et al., 2008). ERDJ5 has been proposed to take part in the processing of non-native disulfide bonds in LDLR which is required for the native disulfide formation and proper folding (Oka et al., 2013). Another member of the PDI family of oxidoreductases, ERP57 is involved in the native disulfide bond formation of substrates in the ER and functions closely with both CNX and CRT (Jessop et al., 2007). ERP57 is indicated to be important for the isomerization of non-native disulfide bonds in LDLR (Jessop et al., 2007). Other PDI family members such as P5 and ERP45 are also reported to exhibit substrate specificity toward LDLR (Jessop et al., 2009).

In addition to the aforementioned chaperones, several private chaperones are involved in LDLR folding. The LBD of LDLR family members require the assistance of the receptor associated protein (RAP) for maturation, which prevents premature interaction of the domain with its ligands in the same compartment (Herz and Marschang, 2003). The BOCA/MESD family of chaperones is shown to be specifically required for the folding of the $\beta$-propeller domain that is contained within the EGF precursor homology region of LDLR (Culi et al., 2004). Calcium has been shown to an absolute requirement for LDLR folding in the ER and lack of calcium, even at very early folding stages, was reported to result in irreversible misfolding of the wild-type protein (Pena et al., 2010). A detailed depiction of LDLR folding is presented in Figure 2.

\section{Proteostasis Components Involved in the Degradation of LDLR Class II Mutants}

It was demonstrated that different Class II mutants of LDLR affecting the LBD (S156L, C176Y, and E207K) and EGF domain (C646Y) were retained in the ER and degraded by a proteasome-dependent pathway in cell lines stably expressing the mutants (Li et al., 2004). One of the first ER factors discovered to be involved in the retention of mutant LDLR was the molecular chaperone GRP78/BiP (Jørgensen et al., 2000). In human liver cells overexpressing the wild-type and mutant receptors (W556S and C646Y), GRP78 strongly interacts with mutant LDLR whereas GRP78-wild type LDLR interaction is weak, suggesting a key role for this chaperone in ER-retention/quality control of class II LDLR mutants (Jørgensen et al., 2000). However, the overexpression of GRP78 was not capable of rescuing the mutants from ER retention. Nevertheless, abundant GRP78 reduces the processing time of newly synthesized wild type LDLR, suggesting GRP78 is critical in protein maturation of wild type LDLR. Other chaperones GRP94, ERP72 (PDIA4), and CNX have also been found to associate with class II mutants (G544V) but not with wild type LDLR (Sørensen et al., 2006). Recently we have also reported that three LDLR class II variants were found to be associated with ER chaperones: GRP78 (BiP), GRP94, the lectin chaperone CNX (Kizhakkedath et al., 2019). In cells overexpressing the G544V mutant, the ER-retention of the mutant was shown to induce ER-stress and activation of UPR as evidenced by the upregulation of mRNAs for GRP78, GRP94, ERP72, attributed to the activity of ER sensors IRE1 and PERK (Sørensen et al., 2006). Apart from its chaperoning activity, GRP94 also has a very specific role in the maturation and stability of wild-type LDLR, as it was shown to protect LDLR from PCSK-mediated degradation (Poirier et al., 2015).

Very little information is available on the ERAD components involved in the substrate recognition, retrotranslocation and degradation of LDLR mutants. Recent results from our lab indicate that the LDLR mutants interact with HRD1 and its partners SEL1L and OS9 (Kizhakkedath et al., 2019). Our results also demonstrated that proteasomal inhibition leads to stabilization of the ER-retained mutants, but had no effect on their folding. Further, inhibitors of ER mannosidase 1 also had a stabilizing effect on the mutants (Kizhakkedath et al., 2019). ER-retained variants of VLDLR, another LDLR family member, were also found to be degraded by the HRD1-SEL1L mediated proteasomal degradation (Kizhakkedath et al., 2018). Unlike VLDLR mutants, the ER retained LDLR mutants were not observed to be aggregation-prone, though overexpression of mutants caused ER stress (Kizhakkedath et al., 2019). The ERAD adaptor protein SEL1L is reported to also play an ERAD independent role in the maturation and processing of lipoprotein lipase (LPL) and hepatic lipid metabolism (Sha et al., 2014). The cell-surface rescue of an ER-retained LDLR mutant was demonstrated to be possible by the use of a chemical chaperone 4-phenylbutyrate (4-PBA) (Tveten et al., 2007). It was later revealed that 4-PBA targets COPII protein and reduces the stringency of ER-retention of misfolded substrates (Ma et al., 2017). It was suggested that stringent ER retention of misfolded substrates requires the efficient packaging of p24-family of proteins via the $\mathrm{B}$ site of the COPII coat and 4-PBA competes with p24 and reduces this stringency (Ma et al., 2017). The available information about the ERAD of misfolded LDLR class II mutants are limited and more detailed investigations utilizing cellular 
models derived from FH patients or model systems expressing physiological levels of LDLR mutants are still required to enhance our understanding of the specificities. However, it is likely that many of the ER factors involved in the folding pathway and physiological quantity control of LDLR participate in some of these processes (Figure 2).

The role of non-canonical ERAD pathways in the degradation of LDLR class II mutants have not been explored to our knowledge. We have reported previously that a small fraction of ER-retained VLDLR missense mutants are aggregation-prone and may undergo an autophagy-related process for degradation (Kizhakkedath et al., 2018). It is probable that aggregation-prone class II LDLR mutants also might undergo non-canonical ERAD.

\section{A Possible Role of ERAD Components Implicated in Misfolded Membrane Proteins in the ERAD of LDLR Mutants}

The mechanisms by which the mutations affecting the TM domain of LDLR cause FH are only emerging. It was reported that a mutation affecting the TM domain of the LDLR (G805R), undergoes ectodomain cleavage by a metalloproteinase in the ER and results in lower LDLR levels at the cell surface (Strøm et al., 2014). The ER-resident rhomboid protease RHBDL4 is proposed as a likely candidate for this metalloproteinase. The cleaved ectodomain however does not undergo proteasomal degradation, instead, appear to pass through the secretory pathway and eventually get secreted to the extracellular space (Strøm et al., 2014). Subsequent studies revealed that many mutations affecting the TM domain of LDLR interfere with membrane-insertion of LDLR are subjected to diverse processes such as metalloproteinase cleavage, complete extracellular secretion or rapid degradation at the cell surface (Strøm et al., 2015). The underlying mechanisms of low cell-surface expression of some of these mutants were elusive (Strøm et al., 2017) and it has been proposed that the mutations affecting the TM domain of LDLR must therefore be considered to be a separate class.

\section{DEREGULATION OF ER HOMEOSTASIS AND ACTIVATION OF UPR BY LDLR MISSENSE MUTANTS}

Accumulation of unfolded proteins in ER activates a battery of cellular stress responses, altogether called as UPR. The UPR aims to restore the normal ER-homeostasis, however, if the stress is severe and irreversible, then UPR switches to apoptosis. Sørensen et al. (2006) for the first time reported that overexpression of LDLR mutants causes ER-stress and elicit UPR (Figure 2). Recent studies from our lab have also confirmed that LDLR mutants retained in ER results in the activation of UPR (Kizhakkedath et al., 2019). However, quite surprisingly, studies focusing on ER-stress, especially the link between ER-stress activation and cellular signaling process that modulate cell fate are missing. These studies are significantly relevant in understanding the molecular pathology of FH where liver damage due to cell death is a critical factor. The following is a detailed picture of the research that has been done pertaining to ER-stress in cell line models expressing mutant LDLR. We also briefly discuss the contradicting findings in stem cell model of FH where ER-stress is not activated when mutant LDLR is expressed.

\section{UPR: An Overview}

Endoplasmic reticulum serves as a site for protein synthesis, folding as well as the internal cellular calcium reservoir and hence plays a critical role in cell physiology. Disruption of ER homeostasis due to derailed calcium physiology; redox imbalance; accumulation of misfolded proteins causes ERstress (Almanza et al., 2019). Cells respond to ER-stress by activating components of counter stress response mechanisms together named UPR. UPR predominantly involves the shutdown of protein translation to reduce further protein load in ER, transcriptional upregulation of ER chaperones to assist protein folding and retrotranslocation of irreversibly misfolded proteins via ERAD. UPR is initially aimed to alleviate ER-stress and regain the normal ER-physiology. However, if the stress persists and the damage is irreversible, then the initial adaptive UPR switches to ER stress-induced apoptosis (Szegezdi et al., 2006; Almanza et al., 2019). UPR is initiated by three major ER stress sensors; PERK, IRE1, and ATF6. The three ER stress sensors are maintained inactive in resting cells by binding to ER chaperone BiP. During ER stress, BiP, which has more affinity to misfolded proteins detaches from the ER sensors and causes the activation of the latter. The concerted cellular response to ER stress is largely mediated by these sensors (Almanza et al., 2019).

Activated PERK phosphorylates the eukaryotic initiation factor 2 alpha (eIF2 alpha) (Schröder and Kaufman, 2005). Phosphorylation of eIF2 blocks cap-dependent translation and thereby reduce further protein load in the ER. Interestingly, certain mRNAs such as ATF4 which possess internal ribosome entry sites at $5^{\prime}$ at their untranslated regions can bypass the PERK-eIF2 alpha pathway mediated translational block. ATF4 up-regulates the expression of ER chaperones as well as induces $\mathrm{CHOP}$, a pro-apoptotic transcriptional factor that induce apoptosis by repressing the anti-apoptotic protein $\mathrm{Bcl}-2$. Initial PERK activation mounts a pro-survival adaptive UPR, however, persistent activation of PERK due to unresolved ER-stress leads to ATF4 mediated transcriptional induction of $\mathrm{CHOP}$ which switches initial adaptive UPR to ER-stress mediated apoptosis. ATF6 is cleaved by two Golgi resident proteases named site- 1 and site-2 to generate an active transcriptional factor which induces the expression of ER chaperones and folding enzymes. Apart from the ER chaperones, ATF6 induces the upregulation of XBP1 mRNA, which is further processed by splicing into a smaller mRNA (XBP1s) by the ribonuclease activity of IRE-1, another ER stress sensor. Similar to ATF-6, the protein encoded by XBP1s is an active transcription factor which induces the expression of ER chaperones, folding enzymes and ERAD components (Figure 2).

Activation of UPR is aimed to resolve the stress in ER and bring back the normal ER homeostasis. Notably, major targets of all three ER stress sensors are directed to mitigate any harmful consequences of ER stress. However, when stress is overwhelming and beyond the capacity of ER adaptive stress machinery then the initial adaptive UPR switches to ER-stress activated apoptosis. 
Persistent activation of three major ER sensors results from unresolvable ER-stress which activates ER-apoptotic signaling principally mediated by pro-apoptotic proteins involving JNK, CHOP and BCL-2 family proteins (Szegezdi et al., 2006). ER-stress induced apoptosis has been implicated as a contributing factor in the pathophysiology of cardiovascular diseases and liver fibrosis (Maiers and Malhi, 2019), two major disease conditions manifested in $\mathrm{FH}$ patients.

\section{ER-Stress and Activation of UPR in Cell Line Models Expressing LDLR Mutants}

Surprisingly, only few studies pertaining to the association of ER-stress with the molecular pathology of $\mathrm{FH}$ have been reported (Jørgensen et al., 2000; Sørensen et al., 2006; Kizhakkedath et al., 2019) in contrast to the fact that around 50\% of LDLR mutations implicated in $\mathrm{FH}$ are class II mutants that are retained in ER due to misfolding. Two decades back, an interesting study by Jorgensen et al. demonstrated ERQC system play a critical role in the proteostasis of class 2 mutant LDLR proteins (Jørgensen et al., 2000). However, it was Sørensen et al. who established for the first time in 2006 that ER-retained LDLR mutants activates ER-stress (Sørensen et al., 2006). Detailed investigations on the activation of UPR by LDLR mutants revealed transcriptional induction of ER chaperones as well as the activation of three UPR sensors (Sørensen et al., 2006).

Our lab has recently reported that two missense LDLR mutants $\mathrm{D} 482 \mathrm{H}$ and $\mathrm{C} 667 \mathrm{~F}$ associated with $\mathrm{FH}$ were misfolded and retained in ER (Kizhakkedath et al., 2019). Further analysis of ER-stress markers in cells expressing the aforesaid mutants pointed activation of UPR. Similar to the previous reports (Jørgensen et al., 2000; Sørensen et al., 2006), we also found ER-retained LDLR mutants strongly interact with GRP78 and other ER chaperones suggesting critical role of these chaperones in ER retention and further ERAD processing of LDLR mutants. Interestingly, our studies disclosed ER-retained LDLR mutants remained soluble in ER lumen which indicates UPR mediated induction of ER chaperones successfully chaperone the mutants and block protein aggregation. However, despite the induction of ER chaperones by UPR, mutant LDLR were not folded and transported to the cell surface. It is well known that ER stress diminish the ERAD capacity of ER and UPR mediated transcriptional induction of ER chaperones is required to sustain the ERAD machinery (Travers et al., 2000). The inferences from ER-stress studies in cell line models overexpressing LDLR mutants suggest that the activation of UPR augment ERAD process where cells can eliminate the unfolded LDLR mutants via ERAD and thereby mitigate toxic ER stress which otherwise activates cell death.

Another important aspect to consider is the role of ER-synthesized sterol regulators such as proprotein convertase subtilisin/kexin type 9 (PCSK9) in the ER physiology of class II LDLR mutant expressing cells. Both PCSK9 and LDLR are transcriptionally upregulated by SREBP2, an ER-resident transcriptional factor that binds to sterol regulatory elements in the promoter regions of sterol inducible genes including LDLR and PCSK9 (Maxwell et al., 2003). PCSK9 is expressed as a pro-form which is autocatalytically processed in ER and the active form is secreted (Seidah et al., 2003). Interestingly, PCSK9 targets surface expressing LDLR for degradation and negatively modulates the latter's function. Decreased LDLR surface expression and increased serum LDL levels have been reported in $\mathrm{FH}$ patients with gain-of-function mutation in PCSK9 (Abifadel et al., 2003; Cameron et al., 2006). In contrast, the African population harboring loss-of-function PCSK9 mutants (unprocessed) have been reported to have lesser occurrence of cardiovascular diseases due to increased expression of surface LDLR and reduced serum LDL levels (Cohen et al., 2005). The aforementioned findings had also raised important questions on how cells manage the ER accumulation of unprocessed PCSK9 mutant pro forms. Does this cause ER-stress? Also, it had been quite intriguing how LDLR and PCSK9 co-exist in the same secretory pathway despite the former being a target of the latter. Hence it was widely speculated that PCSK9 would have interacting protein partners in ER. It was until 2015, Poirier et al. (2015) demonstrated that GRP94, an ER chaperone specifically interacts with PCSK9 and blocks its interaction with LDLR. Interestingly, it was later identified that GRP94 chaperones PCSK mutant pro-forms in ER and alleviate the potential toxic ER-stress (Lebeau et al., 2018). The underlying mechanism was further delineated to GRP94 interaction with mutant PCSK9 which prevents the latter's binding to GRP78, an ER luminal chaperone that signals proteotoxic stress in ER to major ER stress sensors (Lebeau et al., 2018). In a way, contradicting to the blocker role of GRP94 in PCSK9-LDLR interactions, PCSK9 is also reported to act as a chaperone for LDLR. In ER, PCSK9 binds to LDLR and aids the transport of the latter (Stroøm et al., 2014). Interestingly, binding to LDLR augments the autocatalytic processing of PCSK9. However, it is important to note that neither the PCSK9 mutants chaperone LDLR nor the class II LDLR mutants are chaperoned by PCSK9. In fact, reduced levels of PCSK9 has been reported in $\mathrm{FH}$ patients harboring class II LDLR mutants (Cameron et al., 2012). It is yet to be determined how GRP94 modulates PCSK9-LDLR or PCSK9-class II mutant LDLR interactions. It is also worthwhile to investigate the efficiency of PCSK9 processing in hepatocytes homozygous for class II mutant LDLR. The other cardinal question is whether class II mutant LDLR causes PCSK9 pro-form accumulation and consequent ER-stress which further compound the already de-regulated ER homeostasis in $\mathrm{FH}$ patients. It remains largely unknown whether the mutual dependence of these functionally antagonizing proteins (PCSK9 and LDLR) contribute to ER physiology and cholesterol homeostasis in FH patients.

Interestingly, ER stress induced by pharmacological ER stress inducers appear to inhibit the secretion of PCSK9 due to their retention in the ER by GRP94 (Lebeau et al., 2017). This is an interesting finding since LDLR class II mutants are known to induce ER-stress and it remains to be found whether PCSK9 is retained and non-functional in this context. Chemical chaperones or pharmacological chaperones (PCs) have been identified as a promising new strategy to re-instate ER-Golgi-cell surface transport of ER-retained mutant proteins including class II LDLR mutants that retain their original biological function to 
some extent (a detailed review is performed in the coming section "Therapeutic Potential of Pharmacological Chaperones (PCs) and Proteostasis Regulators (PRs) in the Disease Management of FH"). Does PCSK9 remains ER-retained and non-functional when chemical chaperones are used to target LDLR mutants? The absence of functional PCSK9 might be an added advantage as it increases the number of cell surface LDLRs re-instated by the intervention of PCs. Taken together, the activation of ER stress by LDLR mutants and the inhibition of PCSK9 secretion by ER-stress activation can be well exploited for therapeutic management of FH. However, it has to be determined, to what extent ER stress is activated in cells of FH subjects, whether it is in the adaptive range where cellular UPR is equipped to manage the constant levels of ER stress or a severe irreversible ER stress where adaptive UPR response switches to apoptosis.

Also, it is quite important to note that in protein conformation diseases such as alpha-1 antitrypsin deficiency, ER-stress induced apoptosis plays a critical role in the molecular pathology associated with liver failure (Lawless et al., 2004). Adding further, ER-stress has been implicated in the pathology of various diseases such as diabetes, cystic fibrosis and neurodegenerative diseases (Almanza et al., 2019). It remains to be identified whether ER-stress play any role in the pathogenesis of class II mutants associated with FH. In order to establish a link between ER-stress and FH pathology, lymphocytes and fibroblast from FH patients expressing class two LDLR mutants have to be studied for ER-stress activation. It is known that cellular consequence to misfolded proteins retained in ER varies depending on the: mutations, tissue types, between physiological conditions of the same patient (Kim, 1998). It has to be identified whether ER-stress response to various LDLR mutations has any role in the phenotypic variation between $\mathrm{FH}$ patients. It is also an informed presumption that UPR activated in cells expressing mutant LDLR aids cell survival by eliminating the misfolded via ERAD. However, this has to be proved in cells from $\mathrm{FH}$ patients.

\section{ER-Stress Studies in Induced Pluripotent Stem Cells (iPSCs) Model for FH Expressing Class II Mutant LDLR}

Induced Pluripotent Stem Cell (iPSCs) have been developed by reprogramming fibroblasts from a $\mathrm{FH}$ patient carrying a homozygous three-base pair deletion in LDLR exon 4. The mutation results in ER-retention of LDLR and hence comes under class II mutation (Omer et al., 2017). Apart from being a clinically relevant model, class II iPSCs also shows potential for stem cell-based therapy for FH. Genome editing mediated by CRISPR-Cas9 tool successfully corrected the mutation and rescued LDLR function. An interesting recent study by the same group illustrated that FH class II iPSCs and hepatocytes derived from these iPSCs elicit no ER-stress response when LDLR mutants are induced by statins (Omer et al., 2020). Statins are drugs extensively used to reduce serum LDL-cholesterol. They inhibit the enzyme HMG-CoA reductase involved in the synthesis of mevalonate from which the body makes sterols including cholesterol. Statins are known to up-regulate the expression of LDLR. Adding further, the study reveals statin mediated induction of LDLR is higher in FH class II iPSCs compared to the CRISPR corrected ones (Omer et al., 2020). However, the induced mutant LDLR which is trapped in ER elicits no ER-stress response. The report surprisingly contradicts other studies including from our lab where UPR is activated upon the expression of mutant LDLRs (Sørensen et al., 2006; Kizhakkedath et al., 2019). Although ER-stress biology of stem cells is yet to be fully understood, iPSCs are capable of activating UPR in response to pharmacological inducers of ER stress such as tunicamycin. Statins do inhibit UPR in some models, however, lipoprotein deficient serum, which is also known to induce LDLR expression, fails to activate UPR in FH class II iPSCs (Omer et al., 2020). The absence of UPR in response to mutant LDLR accumulation in ER is intriguing and one elementary clarification is that, the amount of induced LDLR mutants falls below the threshold to induce any considerable ER-stress response. It is also possible that the particular mutant used in this study (Omer et al., 2020) is only partially retained in the ER as one can infer from the data where there is a significant presence of the mature form of LDLR in response to statin treatment. The other point to be considered is the efficient removal of mutant LDLR by ERAD and therefore less likely to induce any considerable amount of ER stress. Studies involving analysis of glycosylation status and protein turn over kinetics would clarify whether the accumulated LDLR is completely or partially retained in ER. In cell line overexpressing models, LDLR mutants are expressed from CMV promoters and each cell carries more than one copy of the plasmid and consequently, the protein is expressed in enough quantity to mount an ER-stress response. A comparative protein expression study involving cell line overexpression models and FH class II iPSCs is required to ascertain the above-said assumption.

\section{THERAPEUTIC POTENTIAL OF PHARMACOLOGICAL CHAPERONES (PCs) AND PROTEOSTASIS REGULATORS (PRs) IN THE DISEASE MANAGEMENT OF FH}

Research underpinning the molecular pathology of $\mathrm{FH}$ from various labs including our group have demonstrated that class II mutations in LDLR cause misfolding, ER retention and consequent protein degradation via ERAD (Sørensen et al., 2006; Kizhakkedath et al., 2019). As abundantly mentioned in this review, ER protein quality control systems (ERQC) maintain proteostasis by facilitating protein folding and eliminates misfolded proteins via ERAD. Sophisticated ERQC comprises ER chaperones as well as the protein components of UPR, ERAD and cytosolic proteasomal degradation machinery. Decades-long research in ERQCs led to the development of various proteostasis regulators (PRs) and PCs that either positively or negatively modulates ERQC components (Gámez et al., 2018). Currently, the first-line therapy for FH include statins which either exert their lipid-lowering effect through the inhibition of HMG-CoA reductase or via SREBP activation, which in turn induces LDLR expression. However, SREBP2 also induces the expression of 
PCSK9, which targets LDLR for lysosomal degradation (Dubuc et al., 2004). Therefore, novel targets for the modulation of LDLR expression and function are increasingly being sought as a supplementation therapy with statins.

Multiple reports are available evidencing the successful application of PCs and PRs in various protein conformation diseases (Mu et al., 2008; Mohamed et al., 2017; Gámez et al., 2018). This suggests that PCs and PRs are promising candidates in the clinical management of FH. It is interesting to note that the idea of using PCs or PRs emerged from an early observation where $\triangle F 508$ CFTR, a single phenylalanine deletion mutant found in more than $85 \%$ of cystic fibrosis alleles was functional when expressed in Xenopus oocyte grown at room temperature (Drumm et al., 1991). Normally, $\triangle$ F508 CFTR is misfolded and retained in ER followed by degradation via ERAD. The functional correction of $\triangle F 508$ CFTR at low temperature was later established in vitro in cell lines (Denning et al., 1992). This suggests that restoration of protein transport and functionality of ER-retained mutants can be achieved by modulating proteostasis.

Proteostasis regulators are very often small molecule modulators of protein homeostasis (Balch et al., 2008). PRs predominantly act by manipulating the cellular stress response pathways including UPR. PRs have been proved to rescue misfolded proteins from ER retention either by modulating ERAD or by enhancing the expression of ER/cytosolic chaperones. PRs such as celastrol, curcumin, and HSP90 inhibitors induce the expression of cytosolic chaperones, Kifunensine and Eeyarestatin I inhibit ERAD, thapsigargin modulates calcium signaling and activates UPR (Wang et al., 2011; Gámez et al., 2018).

Unlike PRs, PCs by itself act on the target misfolded proteins and tilt the equilibrium toward the folding state (Ringe and Petsko, 2009). Interestingly, PCs that have been successfully developed for misfolded enzymes are their substrate variants. Substrate binding sites or active binding sites of enzymes are generally formed by more than one domain and therefore PCs bind and aid interaction between protein domains and thereby assist correct folding (Ringe and Petsko, 2009; Gámez et al., 2018). Natural co-factors and ligands are also being used as PCs for various protein conformation diseases. PCs are often protein-specific and sometimes mutation specific. However, cases have been reported where the same PCs are found effective for various mutants of the same protein (Conn and Janovick, 2009). Adding further, the combined application of PRs and PCs can be more effective as PRs increases the cellular chaperonic capability/QCS whereas PCs increases the availability of active folded missense proteins (Gámez et al., 2018).

\section{Potential of PRs and PCs in Treating FH}

The 4-phenyl butyric acid (4-PBA), a low molecular weight bipolar fatty acid derivative appears to rescue ER-retained transport defective class II mutant LDLR G544V in cell line overexpressing model. Although, 4-PBA mediates the rescue of only $30 \%$ of mutant LDLR expressed, the rescued LDLR mutant is expressed on the cell surface and capable of LDL binding and internalization compared to wild type (Tveten et al., 2007). Interestingly, the rescue effect is mutation-specific as other class
II LDLR mutants are not rescued by 4-PBA. Recently, it has been demonstrated that 4-PBA act on the COPII machinery to promote the ER exit of the G544V mutant (Ma et al., 2017). 4-PBA has also been shown to mitigate ER-stress in animal models of neurodegenerative disorders and type 2 diabetes (Özcan et al., 2006; Bondulich et al., 2016). However, despite the promising outcome from the 4-PBA studies, no further studies have been done at preclinical level. It is also notable that very limited studies have been performed regarding the potential of established PRs and PCs in functionally rescuing the class II LDLR mutants.

Our lab has been studying proteostasis regulation of various missense mutants implicated in various genetic diseases including $\mathrm{FH}$. We have demonstrated that the genetic ablation of ERAD components leads to the stabilization of ER-retained VLDLR missense mutants (Kizhakkedath et al., 2018). However, we are yet to demonstrate whether the rescued mutants are functional. Pharmacological inhibitors of ERAD such as Kifunensine and Eeyarestatin I are found to functionally rescue missense mutants associated with lysosomal storage diseases (Wang et al., 2011). Kifunensine inhibits Mannosidase which is a critical component in the recognition of misfolded proteins that are marked for ERAD. Eeyarestatin I blocks the extraction of ubiquitinated proteins from the ER membrane by inhibiting p97 ATPase activity (Wang et al., 2011). Even though we have demonstrated the stabilization of LDLR missense mutants in cell lines (Kizhakkedath et al., 2019), it is yet to be studied whether the aforementioned small molecule ERAD inhibitors have any impacts on the functional rescue of LDLR mutants.

The UPS is the end component of ERAD where the retrotranslocated misfolded proteins are finally processed. We and others have shown that proteasome inhibitors such as MG132 aid protein transport and significantly improve the protein function of missense mutants which otherwise retained in ER and subsequently subjected to ERAD (Wilke et al., 2012; Kizhakkedath et al., 2018). It is quite intriguing how inhibition of proteasome rescues misfolded proteins from ER retention and subsequent ERAD. As reported earlier (Pirkkala et al., 2000), inhibition of proteasome causes perturbation in proteostasis which elicit proteotoxic stress response with transcriptional induction of chaperones. The increase in chaperone reservoir aid protein folding and thus probably explains the partial rescue of ER-retained misfolded proteins and consequent transport to its destined locations. From our studies and other published reports, one can presume that partial functional rescue of ER retained mutant proteins by ERAD blockers and proteasome inhibitors are either mediated by the increased expression of chaperones due to perturbance in proteostasis or the increased boarding of misfolded proteins in ER due to the blocked ERAD. The increased duration of misfolded proteins in the ER lumen might increase their chances to get transported out to Golgi. It would be interesting to investigate these aspects. The exact mechanism by which the inhibition of ERAD or proteasome leads to functional rescue of misfolded proteins is yet to be studied in depth. Proteasome inhibitors such MG132 acts on multiple pathways, the synergistic effect of which has been shown to be protective during acute 
myocardial ischemia (Yu and Kem, 2010). The cardioprotective mechanisms mediated by MG132 were proposed to be through degradation of IkB (inhibitory kB), GRK-2 (G-protein-receptor kinase 2), ARC (apoptosis repressor with caspase recruitment domain), and also by induction of HSP (Yu and Kem, 2010). In HepG2 cells, other than proteasomal inhibition, MG132 has been shown to enhance LDL uptake by upregulating LDLR mRNA expression through a PKC-dependent pathway. An unexpected effect of MG132 was the suppression of PCSK9 expression, which aided in decreased LDLR degradation and enhanced LDL uptake (Yan et al., 2014). Curcumin is a plant-derived natural polyphenolic compound which has been demonstrated to induce HSPs and reported to have anti-inflammatory, antioxidant properties, in addition to preventing protein-aggregation (Maiti et al., 2014). Curcumin has been recently reported to produce a hypocholesterolemic effect by enhancing the cell-surface expression of LDLR and LDL uptake through downregulation of PCSK9 gene expression in HepG2 cells (Tai et al., 2014). The effect of curcumin on the rescue of mutant LDLR misfolding has not been investigated so far and can be explored. As mentioned above, only a few studies have been performed on the potential roles of PCs and PRs in the rescue of class II LDLR mutants. More investigations involving already established PRs and PCs that show rescue potential in other protein conformation diseases might improve the chances of finding effective PCs/PRs for FH class II mutant LDLRs.

\section{Potential Challenges in Using PRs and PCs as Drugs for FH}

Even though, the cell line-based studies are encouraging, targeting normal cellular process such as ERAD and proteasome has deleterious effects. One has to be cautious of the fact that majority of the information on the molecular pathology of class II LDLR mutants come from cell line-based overexpression studies. The cell line-based data has to be validated with studies involving tissues from FH patients. Low efficiency also poses a problem while considering PCs/PRs as therapeutic agents. Cell line-based study shows only $30 \%$ functional recovery of rescued LDLR by 4-PBA, an extensively studied chemical chaperone for various mutants associated with protein conformational diseases. Pre-clinical studies show that application of PCs result in variable increase in the activity of mutant enzymes associated with lysosomal storage diseases (Parenti et al., 2015). Some mutations respond well and others not at all (Parenti et al., 2015). Hence, the efficiency of PCs, in general, is debatable. Therefore, it is a long way ahead to determine whether restoration of mutant LDLR activity by 4 -PBA is beneficial to $\mathrm{FH}$ patients.

It is quite interesting to note that 4-PBA mediated rescue of LDLR mutants is mutation specific. The authors diligently prove that the other mutations in the same domain of the protein which are also class II mutations are not rescued by 4-PBA (Tveten et al., 2007). One can reasonably assume that mutations that grossly affect the protein stability or core LDL binding site may not be rescued by PCs. However, regardless of various limitations, PCs and PRs have the potential to be considered as therapeutic agents for $\mathrm{FH}$ patients with class II LDLR mutations.

\section{CONCLUSION AND FUTURE PERSPECTIVES}

In this review, we have outlined the impact of class II LDLR mutants on ER-proteostasis and how it can be modulated for the therapeutic management of FH. Conventional lipidlowering drugs are effective in maintaining LDL-C levels in heterozygous $\mathrm{FH}$ patients since these patients have residual LDLR function due to the presence of a normal copy of the $L D L R$ gene. In homozygous FH patients where a functional LDLR is lacking, the current LDL-C lowering drugs have minimal effect (Wilemon et al., 2020). A growing body of evidence suggests that ER-proteostasis can be modulated for therapeutic purposes to treat various protein conformation diseases including class II FH. LDLR is the founding member of the LDL receptor family whose members share structural and functional domains. The exquisite structure of these class of proteins requires a specialized array of private and global quality control factors, many of which remain to be unraveled. Despite the significant development in ERAD research, only limited information is available on the factors responsible for the recognition, ER-retention and degradation of defective LDLRs. Notably, we are yet to find out whether non-canonical ERAD pathways such as ER-phagy and microautophagy are involved in the disposal of misfolded mutant LDLRs. Cell line models of class II mutants associated with $\mathrm{FH}$ indicate a role for ER-stress and UPR activation in the pathogenesis. More studies using fibroblasts from heterozygous and homozygous FH patients with diverse Class II LDLR mutations will clarify this aspect and lay foundations for designing therapies focused on adaptive UPR and folding-rescue of these class of mutants. A growing body of evidence suggests that PCs and PRs have the potential to augment conventional therapies for FH. Though the benefits have to be carefully weighed against manipulating the natural processes of ERAD and proteostasis, PRs have the potential to be considered for therapeutic management of FH. Cholesterol is a constituent of the ER membrane and is regulated by ER. Therefore, mechanisms aimed at restoring ER homeostasis are likely to influence cholesterol homeostasis (Fu et al., 2012). Additional investigations on the influence of PRs on cholesterol metabolism independent of proteostasis would also provide insights into novel pathways of LDLR regulation.

\section{AUTHOR CONTRIBUTIONS}

$\mathrm{DO}, \mathrm{PK}$, and $\mathrm{AJ}$ drafted different sections of the manuscript. PK and BA compiled and edited the manuscript. PK and DV created the figures. DO, PK, DV, AJ, and BA critically reviewed and edited the manuscript. All authors contributed to the article and approved the submitted version. 


\section{FUNDING}

The FH project in BA laboratory is supported by grants from UAEU through Zayed Center for Health Sciences (31R243 and 31R232).

\section{REFERENCES}

Abifadel, M., Varret, M., Rabès, J. P., Allard, D., Ouguerram, K., Devillers, M., et al. (2003). Mutations in PCSK9 cause autosomal dominant hypercholesterolemia. Nat. Genet. 34, 154-156. doi: 10.1038/ng1161

Aebi, M., Bernasconi, R., Clerc, S., and Molinari, M. (2010). N-glycan structures: recognition and processing in the ER. Trends Biochem. Sci. 35, 74-82. doi: 10.1016/j.tibs.2009.10.001

Ali, B. R., Ben-Rebeh, I., John, A., Akawi, N. A., Milhem, R. M., Al-Shehhi, N. A., et al. (2011). Endoplasmic reticulum quality control is involved in the mechanism of endoglin-mediated hereditary haemorrhagic telangiectasia. PLoS One 6:e26206. doi: 10.1371/journal.pone.0026206

Al-Kindi, A., Kizhakkedath, P., Xu, H., John, A., Sayegh, A. A. A., Ganesh, A., et al. (2014). A novel mutation in DDR2 causing spondylo-meta-epiphyseal dysplasia with short limbs and abnormal calcifications (SMED-SL) results in defective intra-cellular trafficking. BMC Med. Genet. 15:42. doi: 10.1186/1471-2350$15-42$

Almanza, A., Carlesso, A., Chintha, C., Creedican, S., Doultsinos, D., Leuzzi, B., et al. (2019). Endoplasmic reticulum stress signalling - from basic mechanisms to clinical applications. FEBS J. 286, 241-278. doi: 10.1111/febs.14608

Alonso, R., Andres, E., Mata, N., Fuentes-Jiménez, F., Badimón, L., López-Miranda, J., et al. (2014). Lipoprotein(a) levels in familial hypercholesterolemia: an important predictor of cardiovascular disease independent of the type of LDL receptor mutation. J. Am. Coll. Cardiol. 63, 1982-1989. doi: 10.1016/j.jacc.2014. 01.063

Balch, W. E., Morimoto, R. I., Dillin, A., and Kelly, J. W. (2008). Adapting proteostasis for disease intervention. Science 319, 916-919.

Banerjee, P., Chan, K. C., Tarabocchia, M., Benito-Vicente, A., Alves, A. C., Uribe, K. B., et al. (2019). Functional analysis of LDLR (low-density lipoprotein receptor) variants in patient lymphocytes to assess the effect of evinacumab in homozygous familial hypercholesterolemia patients with a spectrum of LDLR activity. Arterioscler. Thromb Vasc. Biol. 39, 2248-2260. doi: 10.1161/ ATVBAHA.119.313051

Beglova, N., Jeon, H., Fisher, C., and Blacklow, S. C. (2004). Cooperation between fixed and low $\mathrm{pH}$-inducible interfaces controls lipoprotein release by the LDL receptor. Mol. Cell 16, 281-292. doi: 10.1016/j.molcel.2004.09.038

Benito-Vicente, A., Alves, A. C., Etxebarria, A., Medeiros, A. M., Martin, C., and Bourbon, M. (2015). The importance of an integrated analysis of clinical, molecular, and functional data for the genetic diagnosis of familial hypercholesterolemia. Genet. Med. 17, 980-988. doi: 10.1038/gim. 2015.14

Benito-Vicente, A., Uribe, K. B., Jebari, S., Galicia-Garcia, U., Ostolaza, H., and Martin, C. (2018). Validation of LDLr activity as a tool to improve genetic diagnosis of familial hypercholesterolemia: a retrospective on functional characterization of LDLr variants. Int. J. Mol. Sci. 19:1676. doi: 10.3390/ ijms 19061676

Bianchini, E., Fanin, M., Mamchaoui, K., Betto, R., and Sandonà, D. (2014). Unveiling the degradative route of the $\mathrm{V} 247 \mathrm{M}$ a-sarcoglycan mutant responsible for LGMD-2D. Hum. Mol. Genet. 23, 3746-3758. doi: 10.1093/hmg/ ddu088

Bondulich, M. K., Guo, T., Meehan, C., Manion, J., Rodriguez Martin, T., Mitchell, J. C., et al. (2016). Tauopathy induced by low level expression of a human brain-derived tau fragment in mice is rescued by phenylbutyrate. Brain 139, 2290-2306. doi: 10.1093/brain/aww137

Braakman, I., and Hebert, D. N. (2013). Protein folding in the endoplasmic reticulum. Cold Spring Harb. Perspect. Biol. 5:a013201. doi: 10.1101/cshperspect. a013201

Bradley, W. A., and Gianturco, S. H. (1986). ApoE is necessary and sufficient for the binding of large triglyceride-rich lipoproteins to the LDL receptor; apoB is unnecessary. J. Lipid Res. 27, 40-48.

\section{SUPPLEMENTARY MATERIAL}

The Supplementary Material for this article can be found online at: https://www.frontiersin.org/articles/10.3389/fgene. 2020.570355/full\#supplementary-material

Briant, K., Koay, Y. H., Otsuka, Y., and Swanton, E. (2015). ERAD of proteins containing aberrant transmembrane domains requires ubiquitylation of cytoplasmic lysine residues. J. Cell Sci. 128, 4112-4125. doi: 10.1242/jcs. 171215

Brodsky, J. L., and Skach, W. R. (2011). Protein folding and quality control in the endoplasmic reticulum: recent lessons from yeast and mammalian cell systems. Curr. Opin. Cell Biol. 23, 464-475. doi: 10.1016/j.ceb.2011.05.004

Brown, M. S., Goldstein, J. L., and Braakman, I. (1986). A receptor-mediated pathway for cholesterol homeostasis. Science 232, 34-47. doi: 10.1126/science. 3513311

Cameron, J., Bogsrud, M. P., Tveten, K., Strom, T. B., Holven, K., Berge, K. E., et al. (2012). Serum levels of proprotein convertase subtilisin/kexin type 9 in subjects with familial hypercholesterolemia indicate that proprotein convertase subtilisin/kexin type 9 is cleared from plasma by low-density lipoprotein receptor-independent pathways. Transl. Res. 160, 125-130. doi: 10.1016/j.trsl 2012.01.010

Cameron, J., Holla, ØL., Ranheim, T., Kulseth, M. A., Berge, K. E., and Leren, T. P. (2006). Effect of mutations in the PCSK9 gene on the cell surface LDL receptors. Hum. Mol. Genet. 15, 1551-1558. doi: 10.1093/hmg/ddl077

Carvalho, P., Goder, V., and Rapoport, T. A. (2006). Distinct ubiquitin-ligase complexes define convergent pathways for the degradation of ER proteins. Cell 126, 361-373. doi: 10.1016/j.cell.2006.05.043

Christianson, J. C., Olzmann, J. A., Shaler, T. A., Sowa, M. E., Bennett, E. J., Richter, C. M., et al. (2011). Defining human ERAD networks through an integrative mapping strategy. Nat. Cell Biol. 14, 93-105. doi: 10.1038/ncb2383

Christianson, J. C., Shaler, T. A., Tyler, R. E., and Kopito, R. R. (2008). OS-9 and GRP94 deliver mutant $\alpha 1$-antitrypsin to the Hrd1-SEL1L ubiquitin ligase complex for ERAD. Nat. Cell Biol. 10, 272-282. doi: 10.1038/ncb1689

Christianson, J. C., and Ye, Y. (2014). Cleaning up in the endoplasmic reticulum: ubiquitin in charge. Nat. Struct. Mol. Biol. 21, 325-335. doi: 10.1038/nsmb.2793

Cohen, J., Pertsemlidis, A., Kotowski, I. K., Graham, R., Garcia, C. K., and Hobbs, H. H. (2005). Low LDL cholesterol in individuals of African descent resulting from frequent nonsense mutations in PCSK9. Nat. Genet. 37, 161-165. doi: $10.1038 /$ ng1509

Conn, P. M., and Janovick, J. A. (2009). Drug development and the cellular quality control system. Trends Pharmacol. Sci. 30, 228-233. doi: 10.1016/j.tips.2009. 02.002

Culi, J., Springer, T. A., and Mann, R. S. (2004). Boca-dependent maturation of $\beta$ propeller/EGF modules in low-density lipoprotein receptor proteins. EMBO J. 23, 1372-1380. doi: 10.1038/sj.emboj.7600132

Davis, C. G., Goldstein, J. L., Südhof, T. C., Anderson, R. G. W., Russell, D. W., and Brown, M. S. (1987). Acid-dependent ligand dissociation and recycling of LDL receptor mediated by growth factor homology region. Nature 326, 760-765. doi: $10.1038 / 326760 \mathrm{a} 0$

De Leonibus, C., Cinque, L., and Settembre, C. (2019). Emerging lysosomal pathways for quality control at the endoplasmic reticulum. FEBS Lett. 593, 2319-2329. doi: 10.1002/1873-3468.13571

DeBose-Boyd, R. A. (2008). Feedback regulation of cholesterol synthesis: sterolaccelerated ubiquitination and degradation of HMG CoA reductase. Cell Res. 18, 609-621. doi: 10.1038/cr.2008.61

Denning, G. M., Anderson, M. P., Amara, J. F., Marshall, J., Smith, A. E., and Welsh, M. J. (1992). Processing of mutant cystic fibrosis transmembrane conductance regulator is temperature-sensitive. Nature 358, 761-764. doi: 10.1038/358761a0

Drumm, M. L., Wilkinson, D. J., Smit, L. S., Worrell, R. T., Strong, T. V., Frizzell, R. A., et al. (1991). Chloride conductance expressed by $\Delta$ F508 and other mutant CFTRs in xenopus oocytes. Science 254, 1797-1799. doi: 10.1126/ science. 1722350

Dubuc, G., Chamberland, A., Wassef, H., Davignon, J., Seidah, N. G., Bernier, L., et al. (2004). Statins upregulate PCSK9, the gene encoding the proprotein convertase neural apoptosis-regulated convertase-1 implicated in familial 
hypercholesterolemia. Arterioscler. Thromb Vasc. Biol. 24, 1454-1459. doi: 10. 1161/01.ATV.0000134621.14315.43

Esser, V., and Russell, D. W. (1988). Transport-deficient mutations in the low density lipoprotein receptor. Alterations in the cysteine-rich and cysteinepoor regions of the protein block intracellular transport. J. Biol. Chem. 263, 13276-13281.

Etxebarria, A., Benito-Vicente, A., Alves, A. C., Ostolaza, H., Bourbon, M., and Martin, C. (2014). Advantages and versatility of fluorescence-based methodology to characterize the functionality of LDLR and class mutation assignment. PLoS One 9:e112677. doi: 10.1371/journal.pone.0112677

Etxebarria, A., Benito-Vicente, A., Palacios, L., Stef, M., Cenarro, A., Civeira, F., et al. (2015). Functional characterization and classification of frequent lowdensity lipoprotein receptor variants. Hum. Mutat. 36, 129-141. doi: 10.1002/ humu. 22721

Fang, S., Ferrone, M., Yang, C., Jensen, J. P., Tiwari, S., and Weissman, A. M. (2001). The tumor autocrine motility factor receptor, gp 78 , is a ubiquitin protein ligase implicated in degradation from the endoplasmic reticulum. Proc. Natl. Acad. Sci. U.S.A. 98, 14422-14427. doi: 10.1073/pnas. 251401598

Fass, D., Blacklow, S., Kim, P. S., and Berger, J. M. (1997). Molecular basis of familial hypercholesterolaemia from structure of LDL receptor module. Nature 388, 691-693. doi: 10.1038/41798

Feige, M. J., and Hendershot, L. M. (2013). Quality control of integral membrane proteins by assembly-dependent membrane integration. Mol. Cell 51, 297-309. doi: 10.1016/j.molcel.2013.07.013

Fleig, L., Bergbold, N., Sahasrabudhe, P., Geiger, B., Kaltak, L., and Lemberg, M. K. (2012). Ubiquitin-dependent intramembrane rhomboid protease promotes ERAD of membrane proteins. Mol. Cell 47, 558-569. doi: 10.1016/j.molcel.2012. 06.008

Francke, U., Brown, M. S., and Goldstein, J. L. (1984). Assignment of the human gene for the low density lipoprotein receptor to chromosome 19: synteny of a receptor, a ligand, and a genetic disease. Proc. Natl. Acad. Sci. U.S.A. 81, 2826-2830. doi: 10.1073/pnas.81.9.2826

Fregno, I., and Molinari, M. (2019). Proteasomal and lysosomal clearance of faulty secretory proteins: ER-associated degradation (ERAD) and ER-to-lysosomeassociated degradation (ERLAD) pathways. Crit. Rev. Biochem. Mol. Biol. 54, 153-163. doi: 10.1080/10409238.2019.1610351

Fu, S., Watkins, S. M., and Hotamisligil, G. S. (2012). The role of endoplasmic reticulum in hepatic lipid homeostasis and stress signaling. Cell Metab. 15, 623-634. doi: 10.1016/j.cmet.2012.03.007

Galicia-Garcia, U., Benito-Vicente, A., Uribe, K. B., Jebari, S., Larrea-Sebal, A., Alonso-Estrada, R., et al. (2020). Mutation type classification and pathogenicity assignment of sixteen missense variants located in the EGF-precursor homology domain of the LDLR. Sci. Rep. 10:1727. doi: 10.1038/s41598-02058734-9

Gámez, A., Yuste-Checa, P., Brasil, S., Briso-Montiano, A., Desviat, L. R., Ugarte, M., et al. (2018). Protein misfolding diseases: prospects of pharmacological treatment. Clin. Genet. 93, 450-458. doi: 10.1111/cge.13088

Garcia, C. K., Wilund, K., Arca, M., Zuliani, G., Fellin, R., Maioli, M., et al. (2001). Autosomal recessive hypercholesterolemia caused by mutations in a putative LDL receptor adaptor protein. Science 292, 1394-1398. doi: 10.1126/science. 1060458

Gent, J., and Braakman, I. (2004). Low-density lipoprotein receptor structure and folding. Cell Mol. Life Sci. 61, 2461-2470. doi: 10.1007/s00018-004-4090-3

Goldstein, J. L., and Brown, M. S. (1990). Regulation of the mevalonate pathway. Nature 343, 425-430. doi: 10.1038/343425a0

Goldstein, J. L., and Brown, M. S. (2009). The LDL receptor. Arterioscler. Thromb Vasc. Biol. 29, 431-438. doi: 10.1161/ATVBAHA.108.179564

Guerriero, C. J., and Brodsky, J. L. (2012). The delicate balance between secreted protein folding and endoplasmic reticulum-associated degradation in human physiology. Physiol. Rev. 92, 537-576. doi: 10.1152/physrev.00027. 2011

Guo, J., Gao, Y., Li, X., He, Y., Zheng, X., Bi, J., et al. (2019). Systematic prediction of familial hypercholesterolemia caused by low-density lipoprotein receptor missense mutations. Atherosclerosis 281, 1-8. doi: 10.1016/j.atherosclerosis. 2018.12.003

Hegde, R. S., and Ploegh, H. L. (2010). Quality and quantity control at the endoplasmic reticulum. Curr. Opin. Cell Biol. 22, 437-446. doi: 10.1016/j.ceb. 2010.05.005
Henderson, R., O'Kane, M., McGilligan, V., and Watterson, S. (2016). The genetics and screening of familial hypercholesterolaemia. J. Biomed. Sci. 23:39. doi: 10.1186/s12929-016-0256-1

Herz, J., and Marschang, P. (2003). Coaxing the LDL receptor family into the fold. Cell 112, 289-292.

Hobbs, H. H., Brown, M. S., and Goldstein, J. L. (1992). Molecular genetics of the LDL receptor gene in familial hypercholesterolemia. Hum. Mutat. 1, 445-466. doi: 10.1002/humu. 1380010602

Holst, H. U., Dagnaes-Hansen, F., Corydon, T. J., Andreasen, P. H., Jørgensen, M. M., Kølvraa, S., et al. (2001). LDL receptor-GFP fusion proteins: new tools for the characterisation of disease-causing mutations in the LDL receptor gene. Eur. J. Hum. Genet. 9, 815-822. doi: 10.1038/sj.ejhg.5200718

Horimoto, S., Ninagawa, S., Okada, T., Koba, H., Sugimoto, T., Kamiya, Y., et al. (2013). The unfolded protein response transducer ATF6 represents a novel transmembrane-type endoplasmic reticulum-associated degradation substrate requiring both mannose trimming and SEL1L protein. J. Biol. Chem. 288, 31517-31527. doi: 10.1074/jbc.M113.476010

Hosokawa, N., Wada, I., Nagasawa, K., Moriyama, T., Okawa, K., and Nagata, K. (2008). Human XTP3-B forms an endoplasmic reticulum quality control scaffold with the HRD1-SEL1L ubiquitin ligase complex and BiP. J. Biol. Chem. 283, 20914-20924. doi: 10.1074/jbc.M709336200

Houck, S. A., Ren, H. Y., Madden, V. J., Bonner, J. N., Conlin, M. P., Janovick, J. A., et al. (2014). Quality control autophagy degrades soluble ERAD-resistant conformers of the misfolded membrane protein GnRHR. Mol. Cell 54, 166-179. doi: 10.1016/j.molcel.2014.02.025

Hume, A. N., Buttgereit, J., Al-Awadhi, A. M., Al-Suwaidi, S. S., John, A., Bader, M., et al. (2009). Defective cellular trafficking of missense NPR-B mutants is the major mechanism underlying acromesomelic dysplasia-type Maroteaux. Hum. Mol. Genet. 18, 267-277. doi: 10.1093/hmg/ddn354

Ikonen, E. (2008). Cellular cholesterol trafficking and compartmentalization. Nat. Rev. Mol. Cell Biol. 9, 125-138. doi: 10.1038/nrm2336

Innerarity, T. L., Mahley, R. W., Weisgraber, K. H., Bersot, T. P., Krauss, R. M., Vega, G. L., et al. (1990). Familial defective apolipoprotein B-100: a mutation of apolipoprotein B that causes hypercholesterolemia. J. Lipid Res. 31, $1337-1349$.

Jansens, A., Van Duijn, E., and Braakman, I. (2002). Coordinated nonvectorial folding in a newly synthesized multidomain protein. Science 298, 2401-2403. doi: $10.1126 /$ science. 1078376

Jeenduang, N., Ruangpracha, A., Promptmas, C., Pongrapeeporn, K. U. S., and Porntadavity, S. (2010). Two novel D151Y and M391T LDLR mutations causing LDLR transport defects in Thai patients with Familial hypercholesterolemia. Clin. Chim. Acta 411, 1656-1661. doi: 10.1016/j.cca.2010.06.021

Jeon, H., Meng, W., Takagi, J., Eck, M. J., Springer, T. A., and Blacklow, S. C. (2001). Implications for familial hypercholesterolemia from the structure of the LDL receptor YWTD-EGF domain pair. Nat. Struct. Biol. 8, 499-504. doi: $10.1038 / 88556$

Jessop, C. E., Chakravarthi, S., Garbi, N., Hämmerling, G. J., Lovell, S., and Bulleid, N. J. (2007). ERp57 is essential for efficient folding of glycoproteins sharing common structural domains. EMBO J. 26, 28-40. doi: 10.1038/sj.emboj. 7601505

Jessop, C. E., Watkins, R. H., Simmons, J. J., Tasab, M., and Bulleid, N. J. (2009). Protein disulphide isomerase family members show distinct substrate specificity: P5 is targeted to BiP client proteins. J. Cell Sci. 122, 4287-4295. doi: $10.1242 /$ jcs. 059154

Jiang, L., Wu, W. F., Sun, L. Y., Chen, P. P., Wang, W., Benito-Vicente, A., et al. (2016). The use of targeted exome sequencing in genetic diagnosis of young patients with severe hypercholesterolemia. Sci. Rep. 6:36823. doi: 10. 1038/srep36823

Jo, Y., Lee, P. C. W., Sguigna, P. V., and DeBose-Boyd, R. A. (2011). Sterol-induced degradation of HMG CoA reductase depends on interplay of two Insigs and two ubiquitin ligases, gp78 and Trc8. Proc. Natl. Acad. Sci. U.S.A. 108, 20503-20508. doi: $10.1073 /$ pnas. 1112831108

John, A., Kizhakkedath, P., Al-Gazali, L., and Ali, B. R. (2015). Defective cellular trafficking of the bone morphogenetic protein receptor type II by mutations underlying familial pulmonary arterial hypertension. Gene 561, 148-156. doi: 10.1016/j.gene.2015.02.038

Jørgensen, M. M., Jensen, O. N., Holst, H. U., Hansen, J. J., Corydon, T. J., Bross, P., et al. (2000). Grp78 is involved in retention of mutant low density lipoprotein 
receptor protein in the endoplasmic reticulum. J. Biol. Chem. 275, 33861-33868. doi: 10.1074/jbc.M004663200

Karagöz, G. E., Acosta-Alvear, D., and Walter, P. (2019). The unfolded protein response: detecting and responding to fluctuations in the protein-folding capacity of the endoplasmic reticulum. Cold Spring Harb. Perspect. Biol. 11:a033886. doi: 10.1101/cshperspect.a033886

Kawaguchi, A., Miyatake, K., Yutani, C., Beppu, S., Tsushima, M., Yamamura, T., et al. (1999). Characteristic cardiovascular manifestation in homozygous and heterozygous familial hypercholesterolemia. Am. Heart J. 137, 410-418. doi: 10.1016/S0002-8703(99)70485-0

Khaminets, A., Heinrich, T., Mari, M., Grumati, P., Huebner, A. K., Akutsu, M., et al. (2015). Regulation of endoplasmic reticulum turnover by selective autophagy. Nature 522, 354-358. doi: 10.1038/nature14498

Kim, P. S. (1998). Endocrinopathies in the family of endoplasmic reticulum (ER) storage diseases: disorders of protein trafficking and the role of ER molecular chaperones. Endocr. Rev. 19, 173-202. doi: 10.1210/er.19.2.173

Kitahara, H., Mori, N., Saito, Y., Nakayama, T., Fujimoto, Y., and Kobayashi, Y. (2019). Prevalence of Achilles tendon xanthoma and familial hypercholesterolemia in patients with coronary artery disease undergoing percutaneous coronary intervention. Heart Vessels 34, 1595-1599. doi: 10.1007/s00380-019-01400-6

Kizhakkedath, P., John, A., Al-Gazali, L., and Ali, B. R. (2018). Degradation routes of trafficking-defective VLDLR mutants associated with Dysequilibrium syndrome. Sci. Rep. 8:1583. doi: 10.1038/s41598-017-19053-8

Kizhakkedath, P., John, A., Al-Sawafi, B. K., Al-Gazali, L., and Ali, B. R. (2019). Endoplasmic reticulum quality control of LDLR variants associated with familial hypercholesterolemia. FEBS Open Biol. 9, 1994-2005. doi: 10.1002/ 2211-5463.12740

Kizhakkedath, P., Loregger, A., John, A., Bleijlevens, B., Al-Blooshi, A. S., Al-Hosani, A. H., et al. (2014). Impaired trafficking of the very low density lipoprotein receptor caused by missense mutations associated with dysequilibrium syndrome. Biochim. Biophys. Acta Mol. Cell Res. 1843, 2871-2877. doi: 10.1016/j.bbamcr.2014.08.013

Klee, E. W., and Zimmermann, M. T. (2019). Molecular modeling of LDLR aids interpretation of genomic variants. J. Mol. Med. 97, 533-540. doi: 10.1007/ s00109-019-01755-3

Klose, G., Laufs, U., März, W., and Windler, E. (2014). Familial hypercholesterolemia: developments in diagnosis and treatment. Dtsch. Arztebl. Int. 111, 523-529. doi: 10.3238/arztebl.2014.0523

Koivisto, U. M., Hubbard, A. L., and Mellman, I. (2001). A novel cellular phenotype for familial hypercholesterolemia due to a defect in polarized targeting of LDL receptor. Cell 105, 575-585. doi: 10.1016/S0092-8674(01)00371-3

Lamriben, L., Graham, J. B., Adams, B. M., and Hebert, D. N. (2016). N-Glycanbased ER molecular chaperone and protein quality control system: the calnexin binding cycle. Traffic 17, 308-326. doi: 10.1111/tra.12358

Lawless, M. W., Greene, C. M., Mulgrew, A., Taggart, C. C., O’Neill, S. J., and McElvaney, N. G. (2004). Activation of endoplasmic reticulum-specific stress responses associated with the conformational disease $\mathrm{Z} \alpha 1$-antitrypsin deficiency. J. Immunol. 172, 5722-5726. doi: 10.4049/jimmunol.172.9.5722

Lebeau, P., Al-Hashimi, A., Sood, S., Lhoták, Š, Yu, P., Gyulay, G., et al. (2017). Endoplasmic reticulum stress and $\mathrm{Ca} 2+$ depletion differentially modulate the sterol regulatory protein PCSK9 to control lipid metabolism. J. Biol. Chem. 292, 1510-1523. doi: 10.1074/jbc.M116.744235

Lebeau, P., Platko, K., Al-Hashimi, A. A., Byun, J. H., Lhoták, Š, Holzapfel, N., et al. (2018). Loss-of-function PCSK9 mutants evade the unfolded protein response sensor GRP78 and fail to induce endoplasmic reticulum stress when retained. J. Biol. Chem. 293, 7329-7343. doi: 10.1074/jbc.RA117.001049

Lee, J. H., Lee, G. Y., Jang, H., Choe, S. S., Koo, S. H., and Kim, J. B. (2014). Ring finger protein 20 regulates hepatic lipid metabolism through protein kinase A-dependent sterol regulatory element binding protein $1 \mathrm{c}$ degradation. Hepatology 60, 844-857. doi: 10.1002/hep.27011

Li, Y., Lu, W., Schwartz, A. L., and Bu, G. (2004). Degradation of the LDL receptor class 2 mutants is mediated by a proteasome-dependent pathway. J. Lipid Res. 45, 1084-1091. doi: 10.1194/jlr.M300482-JLR200

Lilley, B. N., and Ploegh, H. L. (2005). Multiprotein complexes that link dislocation, ubiquitination, and extraction of misfolded proteins from the endoplasmic reticulum membrane. Proc. Natl. Acad. Sci. U.S.A. 102, 14296-14301. doi: 10. 1073/pnas.0505014102
Loregger, A., Cook, E. C. L., Nelson, J. K., Moeton, M., Sharpe, L. J., Engberg, S., et al. (2015). A MARCH6 and IDOL E3 ubiquitin ligase circuit uncouples cholesterol synthesis from lipoprotein uptake in hepatocytes. Mol. Cell Biol. 36, 285-294. doi: 10.1128/mcb.00890- 15

Ma, W., Goldberg, E., and Goldberg, J. (2017). ER retention is imposed by COPII protein sorting and attenuated by 4-phenylbutyrate. eLife 6:e26624. doi: 10 . 7554/eLife. 26624

Maiers, J. L., and Malhi, H. (2019). Endoplasmic reticulum stress in metabolic liver diseases and hepatic fibrosis. Semin. Liver Dis. 39, 235-248. doi: 10.1055/ s-0039-1681032

Maiti, P., Manna, J., Veleri, S., and Frautschy, S. (2014). Molecular chaperone dysfunction in neurodegenerative diseases and effects of curcumin. Biomed. Res. Int. 2014:495091. doi: 10.1155/2014/495091

Maxwell, K. N., Soccio, R. E., Duncan, E. M., Sehayek, E., and Breslow, J. L. (2003). Novel putative SREBP and LXR target genes identified by microarray analysis in liver of cholesterol-fed mice. J. Lipid Res. 44, 2109-2119. doi: 10.1194/jlr. M300203-JLR200

Menzies, S. A., Volkmar, N., van den Boomen, D. J. H., Timms, R. T., Dickson, A. S., Nathan, J. A., et al. (2018). The sterol-responsive RNF145 E3 ubiquitin ligase mediates the degradation of HMG-CoA reductase together with gp78 and hrd1. eLife 7:e40009. doi: 10.7554/eLife.40009

Mohamed, F. E., Al-Gazali, L., Al-Jasmi, F., and Ali, B. R. (2017). Pharmaceutical chaperones and proteostasis regulators in the therapy of lysosomal storage disorders: current perspective and future promises. Front. Pharmacol. 8:448. doi: 10.3389/fphar.2017.00448

Mu, T.-W., Ong, D. S. T., Wang, Y.-J., Balch, W. E., Yates, J. R., Segatori, L., et al. (2008). Proteostasis regulators and pharmacologic chaperones synergize to correct protein misfolding diseases. Cell 134, 769-781. doi: 10.1016/j.cell.2008. 06.037

Müller, C. (1938). Xanthomata, hypercholesterolemia, angina pectoris. Acta Med. Scand. 95, 75-84. doi: 10.1111/j.0954-6820.1938.tb19279.x

Nadav, E., Shmueli, A., Barr, H., Gonen, H., Ciechanover, A., and Reiss, Y. (2003). A novel mammalian endoplasmic reticulum ubiquitin ligase homologous to the yeast Hrd1. Biochem. Biophys. Res. Commun. 303, 91-97. doi: 10.1016/S0006291X(03)00279-1

Ni, M., and Lee, A. S. (2007). ER chaperones in mammalian development and human diseases. FEBS Lett. 581, 3641-3651. doi: 10.1016/j.febslet.2007.04.045

Nordestgaard, B. G., Chapman, M. J., Humphries, S. E., Ginsberg, H. N., Masana, L., Descamps, O. S., et al. (2013). Familial hypercholesterolaemia is underdiagnosed and undertreated in the general population: guidance for clinicians to prevent coronary heart disease. Eur. Heart J. 34, 3478-3490a. doi: 10.1093/eurheartj/eht273

Oka, O. B. V., Pringle, M. A., Schopp, I. M., Braakman, I., and Bulleid, N. J. (2013). ERdj5 is the ER reductase that catalyzes the removal of non-native disulfides and correct folding of the LDL receptor. Mol. Cell 50, 793-804. doi: 10.1016/j.molcel.2013.05.014

Okuda-Shimizu, Y., and Hendershot, L. M. (2007). Characterization of an ERAD pathway for nonglycosylated BiP substrates, which require Herp. Mol. Cell 28, 544-554. doi: 10.1016/j.molcel.2007.09.012

Olzmann, J. A., Kopito, R. R., and Christianson, J. C. (2013). The mammalian endoplasmic reticulum-associated degradation system. Cold Spring Harb. Perspect. Biol. 5:a013185. doi: 10.1101/cshperspect.a013185

Omer, L., Hindi, L., Militello, G., Stivers, K. B., Tien, K. C., and Boyd, N. L. (2020). Familial hypercholesterolemia class II low-density lipoprotein receptor response to statin treatment. Dis. Model. Mech. 13:dmm042911. doi: 10.1242/ dmm.042911

Omer, L., Hudson, E. A., Zheng, S., Hoying, J. B., Shan, Y., and Boyd, N. L. (2017). CRISPR correction of a homozygous low-density lipoprotein receptor mutation in familial hypercholesterolemia induced pluripotent stem cells. Hepatol. Commun. 1, 886-898. doi: 10.1002/hep4.1110

Özcan, U., Yilmaz, E., Özcan, L., Furuhashi, M., Vaillancourt, E., Smith, R. O., et al. (2006). Chemical chaperones reduce ER stress and restore glucose homeostasis in a mouse model of type 2 diabetes. Science 313, 1137-1140. doi: 10.1126/ science. 1128294

Parenti, G., Andria, G., and Valenzano, K. J. (2015). Pharmacological chaperone therapy: preclinical development, clinical translation, and prospects for the treatment of lysosomal storage disorders. Mol. Ther. 23, 1138-1148. doi: 10. $1038 / \mathrm{mt} .2015 .62$ 
Pena, F., Jansens, A., Van Zadelhoff, G., and Braakman, I. (2010). Calcium as a crucial cofactor for low density lipoprotein receptor folding in the endoplasmic reticulum. J. Biol. Chem. 285, 8656-8664. doi: 10.1074/jbc.M110.105718

Pirkkala, L., Alastalo, T.-P., Zuo, X., Benjamin, I. J., and Sistonen, L. (2000). Disruption of heat shock factor 1 reveals an essential role in the ubiquitin proteolytic pathway. Mol. Cell Biol. 20, 2670-2675. doi: 10.1128/mcb.20.8.26702675.2000

Poirier, S., Mamarbachi, M., Chen, W. T., Lee, A. S. S., and Mayer, G. (2015). GRP94 regulates circulating cholesterol levels through blockade of PCSK9-induced LDLR degradation. Cell Rep. 13, 2064-2071. doi: 10.1016/j.celrep.2015.11.006

Printsev, I., Curiel, D., and Carraway, K. L. (2017). Membrane protein quantity control at the endoplasmic reticulum. J. Membr. Biol. 250, 379-392. doi: 10. 1007/s00232-016-9931-0

Ringe, D., and Petsko, G. A. (2009). What are pharmacological chaperones and why are they interesting? J. Biol. 8:80. doi: 10.1186/jbiol186

Röhrl, C., and Stangl, H. (2018). Cholesterol metabolism-physiological regulation and pathophysiological deregulation by the endoplasmic reticulum. Wiener Med. Wochenschrift 168, 280-285. doi: 10.1007/s10354-018-0626-2

Rudenko, G., Henry, L., Henderson, K., Ichtchenko, K., Brown, M. S., Goldstein, J. L., et al. (2002). Structure of the LDL receptor extracellular domain at endosomal pH. Science 298, 2353-2358. doi: 10.1126/science.1078124

Ruggiano, A., Foresti, O., and Carvalho, P. (2014). ER-associated degradation: protein quality control and beyond. J. Cell Biol. 204, 869-879. doi: 10.1083/jcb. 201312042

Russell, D. W., Schneider, W. J., Yamamoto, T., Luskey, K. L., Brown, M. S., and Goldstein, J. L. (1984). Domain map of the LDL receptor: sequence homology with the epidermal growth factor precursor. Cell 37, 577-585. doi: 10.1016/ 0092-8674(84)90388-X

Schaefer, J. R., Kurt, B., Sattler, A., Klaus, G., and Soufi, M. (2012). Pharmacogenetic aspects in familial hypercholesterolemia with the special focus on FHMarburg (FH p.W556R). Clin. Res. Cardiol. Suppl. 7, 2-6. doi: 10.1007/s11789-0120041-y

Schröder, M., and Kaufman, R. J. (2005). The mammalian unfolded protein response. Annu. Rev. Biochem. 74, 739-789. doi: 10.1146/annurev.biochem.73. 011303.074134

Seidah, N. G., Benjannet, S., Wickham, L., Marcinkiewicz, J., Bélanger Jasmin, S., Stifani, S., et al. (2003). The secretory proprotein convertase neural apoptosis-regulated convertase 1 (NARC-1): liver regeneration and neuronal differentiation. Proc. Natl. Acad. Sci. U.S.A. 100, 928-933. doi: 10.1073/pnas. 0335507100

Sha, H., Sun, S., Francisco, A. B., Ehrhardt, N., Xue, Z., Liu, L., et al. (2014). The ER-associated degradation adaptor protein Sel1L regulates LPL secretion and lipid metabolism. Cell Metab. 20, 458-470. doi: 10.1016/j.cmet.2014.06.015

Sharpe, L. J., Cook, E. C. L., Zelcer, N., and Brown, A. J. (2014). The UPS and downs of cholesterol homeostasis. Trends Biochem. Sci. 39, 527-535. doi: 10.1016/j.tibs. 2014.08.008

Shenkman, M., and Lederkremer, G. Z. (2019). Compartmentalization and selective tagging for disposal of misfolded glycoproteins. Trends Biochem. Sci. 44, 827-836. doi: 10.1016/j.tibs.2019.04.012

Shu, H., Chi, J., Li, J., Zhang, W., Lv, W., Wang, J., et al. (2017). A novel indel variant in LDLR responsible for familial hypercholesterolemia in a Chinese family. PLoS One 12:e0189316. doi: 10.1371/journal.pone.0189316

Smith, M. H., Ploegh, H. L., and Weissman, J. S. (2011). Road to ruin : targeting proteins for degradation in the endoplasmic reticulum E3 ubiquitin ligases: central organizers. Science 334, 1086-1090.

Sørensen, S., Ranheim, T., Bakken, K. S., Leren, T. P., and Kulseth, M. A. (2006). Retention of mutant low density lipoprotein receptor in endoplasmic reticulum (ER) leads to ER stress. J. Biol. Chem. 281, 468-476. doi: 10.1074/jbc. M507071200

Soutar, A. K., and Naoumova, R. P. (2007). Mechanisms of disease: genetic causes of familial hypercholesterolemia. Nat. Clin. Pract. Cardiovasc. Med. 4, 214-225. doi: $10.1038 /$ ncpcardio0836

Springer, T. A. (1998). An extracellular $\beta$-propeller module predicted in lipoprotein and scavenger receptors, tyrosine kinases, epidermal growth factor precursor, and extracellular matrix components. J. Mol. Biol. 283, 837-862. doi: 10.1006/ jmbi.1998.2115

Stenson, P. D., Mort, M., Ball, E. V., Evans, K., Hayden, M., Heywood, S., et al. (2017). The human gene mutation database: towards a comprehensive repository of inherited mutation data for medical research, genetic diagnosis and next-generation sequencing studies. Hum. Genet. 136, 665-677. doi: 10. 1007/s00439-017-1779-6

Strøm, T. B., Laerdahl, J. K., and Leren, T. P. (2015). Mutation p.L799R in the LDLR, which affects the transmembrane domain of the LDLR, prevents membrane insertion and causes secretion of the mutant LDLR. Hum. Mol. Genet. 24, 5836-5844. doi: 10.1093/hmg/ddv304

Strøm, T. B., Laerdahl, J. K., and Leren, T. P. (2017). Mutations affecting the transmembrane domain of the LDL receptor: impact of charged residues on the membrane insertion. Hum. Mol. Genet. 26, 1634-1642. doi: 10.1093/hmg/ ddx068

Strøm, T. B., Tveten, K., Laerdahl, J. K., and Leren, T. P. (2014). Mutation G805R in the transmembrane domain of the LDL receptor gene causes familial hypercholesterolemia by inducing ectodomain cleavage of the LDL receptor in the endoplasmic reticulum. FEBS Open Biol. 4, 321-327. doi: 10.1016/j.fob. 2014.03.007

Stroøm, T. B., Tveten, K., and Leren, T. P. (2014). Pcsk9 acts as a chaperone for the ldl receptor in the endoplasmic reticulum. Biochem. J. 457, 99-105. doi: 10.1042/BJ20130930

Südhof, T. C., Russell, D. W., Goldstein, J. L., Brown, M. S., Sanchez-Pescador, R., and Bell, G. I. (1985). Cassette of eight exons shared by genes for LDL receptor and EGF precursor. Science 228, 893-895. doi: 10.1126/science.387 3704

Sun, Z., and Brodsky, J. L. (2019). Protein quality control in the secretory pathway. J. Cell Biol. 218, 3171-3187. doi: 10.1083/jcb.201906047

Sundqvist, A., Bengoechea-Alonso, M. T., Ye, X., Lukiyanchuk, V., Jin, J., Harper, J. W., et al. (2005). Control of lipid metabolism by phosphorylation-dependent degradation of the SREBP family of transcription factors by SCFFbw7. Cell Metab. 1, 379-391. doi: 10.1016/j.cmet.2005. 04.010

Susan-Resiga, D., Girard, E., Kiss, R. S., Essalmani, R., Hamelin, J., Asselin, M. C., et al. (2017). The proprotein convertase subtilisin/kexin type 9-resistant R410S low density lipoprotein receptor mutation: a novel mechanism causing familial hypercholesterolemia. J. Biol. Chem. 292, 1573-1590. doi: 10.1074/jbc.M116. 769430

Szegezdi, E., Logue, S. E., Gorman, A. M., and Samali, A. (2006). Mediators of endoplasmic reticulum stress-induced apoptosis. EMBO Rep. 7, 880-885. doi: 10.1038/sj.embor.7400779

Tai, M. H., Chen, P. K., Chen, P. Y., Wu, M. J., Ho, C. T., and Yen, J. H. (2014). Curcumin enhances cell-surface LDLR level and promotes LDL uptake through downregulation of PCSK9 gene expression in HepG2 cells. Mol. Nutr. Food Res. 58, 2133-2145. doi: 10.1002/mnfr.201400366

Travers, K. J., Patil, C. K., Wodicka, L., Lockhart, D. J., Weissman, J. S., and Walter, P. (2000). Functional and genomic analyses reveal an essential coordination between the unfolded protein response and ER-associated degradation. Cell 101, 249-258. doi: 10.1016/S0092-8674(00)80835-1

Tveten, K., Holla, ØL., Ranheim, T., Berge, K. E., Leren, T. P., and Kulseth, M. A. (2007). 4-Phenylbutyrate restores the functionality of a misfolded mutant lowdensity lipoprotein receptor. FEBS J. 274, 1881-1893. doi: 10.1111/j.1742-4658. 2007.05735.x

Ushioda, R., Hoseki, J., Araki, K., Jansen, G., Thomas, D. Y., and Nagata, K. (2008). ERdj5 is required as a disulfide reductase for degradation of misfolded proteins in the ER. Science 321, 569-572. doi: 10.1126/science.1159293

Ushioda, R., Hoseki, J., and Nagata, K. (2013). Glycosylation-independent ERAD pathway serves as a backup system under ER stress. Mol. Biol. Cell 24, 3155-3163. doi: 10.1091/mbc.E13-03-0138

Van Hoof, D., Rodenburg, K. W., and Van der Horst, D. J. (2005). Intracellular fate of LDL receptor family members depends on the cooperation between their ligand-binding and EGF domains. J. Cell Sci. 118(Pt 6), 1309-1320. doi: $10.1242 /$ jcs. 01725

Varret, M., and Rabès, J.-P. (2012). Missense mutation in the LDLR gene: a wide spectrum in the severity of familial hypercholesterolemia. Mutations Hum. Genet. Dis. 55-74. doi: 10.5772/2912

Vembar, S. S., and Brodsky, J. L. (2008). One step at a time: endoplasmic reticulumassociated degradation. Nat. Rev. Mol. Cell Biol. 9, 944-957. doi: 10.1038/ nrm 2546

Wang, F., Song, W., Brancati, G., and Segatori, L. (2011). Inhibition of endoplasmic reticulum-associated degradation rescues native folding in loss of function 
protein misfolding diseases. J. Biol. Chem. 286, 43454-43464. doi: 10.1074/jbc. M111.274332

Wang, H., Xu, S., Sun, L., Pan, X., Yang, S., and Wang, L. (2014). Functional characterization of two low-density lipoprotein receptor gene mutations in two Chinese patients with familial hypercholesterolemia. PLoS One 9:e92703. doi: 10.1371/journal.pone.0092703

Ward, C. L., Omura, S., and Kopito, R. R. (1995). Degradation of CFTR by the ubiquitin-proteasome pathway. Cell 83, 121-127. doi: 10.1016/0092-8674(95) 90240-6

Wiegman, A., Gidding, S. S., Watts, G. F., Chapman, M. J., Ginsberg, H. N., Cuchel, M., et al. (2015). Familial hypercholesterolæmia in children and adolescents: gaining decades of life by optimizing detection and treatment. Eur. Heart J. 36, 2425-2437. doi: 10.1093/eurheartj/ehv157

Wilemon, K. A., Patel, J., Aguilar-Salinas, C., Ahmed, C. D., Alkhnifsawi, M., Almahmeed, W., et al. (2020). Reducing the clinical and public health burden of familial hypercholesterolemia: a global call to action. JAMA Cardiol. 5, 217-229. doi: 10.1001/jamacardio.2019.5173

Wilke, M., Bot, A., Jorna, H., Scholte, B. J., and de Jonge, H. R. (2012). Rescue of murine F508del CFTR activity in native intestine by low temperature and proteasome inhibitors. PLoS One 7:e52070. doi: 10.1371/journal.pone.005 2070

Yamamoto, T., Davis, C. G., Brown, M. S., Schneider, W. J., Casey, M. L., Goldstein, J. L., et al. (1984). The human LDL receptor: a cysteine-rich protein with multiple Alu sequences in its mRNA. Cell 39, 27-38. doi: 10.1016/00928674(84)90188-0
Yan, H., Ma, Y. L., Gui, Y. Z., Wang, S. M., Wang, X. B., Gao, F., et al. (2014). MG132, a proteasome inhibitor, enhances LDL uptake in HepG2 cells in vitro by regulating LDLR and PCSK9 expression. Acta Pharmacol. Sin. 35, 994-1004. doi: 10.1038/aps.2014.52

Yu, X., and Kem, D. C. (2010). Proteasome inhibition during myocardial infarction. Cardiovasc. Res. 85, 312-320. doi: 10.1093/cvr/cvp309

Zapun, A., Darby, N. J., Tessier, D. C., Michalak, M., Bergeron, J. J. M., and Thomas, D. Y. (1998). Enhanced catalysis of ribonuclease B folding by the interaction of calnexin or calreticulin with ERp57. J. Biol. Chem. 273, 6009-6012. doi: 10.1074/jbc.273.11.6009

Zelcer, N., Hong, C., Boyadjian, R., and Tontonoz, P. (2009). LXR regulates cholesterol uptake through idol-dependent ubiquitination of the LDL receptor. Science 325, 100-104. doi: 10.1126/science.1168974

Conflict of Interest: The authors declare that the research was conducted in the absence of any commercial or financial relationships that could be construed as a potential conflict of interest.

Copyright (c) 2020 Oommen, Kizhakkedath, Jawabri, Varghese and Ali. This is an open-access article distributed under the terms of the Creative Commons Attribution License (CC BY). The use, distribution or reproduction in other forums is permitted, provided the original author(s) and the copyright owner(s) are credited and that the original publication in this journal is cited, in accordance with accepted academic practice. No use, distribution or reproduction is permitted which does not comply with these terms. 